Electronic Supporting information for

\title{
Unravelling the Role of Excess Ligand in Nanoparticle Pattern Formation from an Evaporatively Dewetting Nanofluid Droplet
}

\author{
Kaustav Bhattacharjee, ${ }^{* 1}$ Korak Biswas, ${ }^{2}$ and Bhagavatula L. V. Prasad ${ }^{81,3}$ \\ ${ }^{1}$ Physical and Material Chemistry Division, National Chemical Laboratory, Pune 411008, India \\ ${ }^{2}$ Department of Physics, Indian Institute of Science Education and Research, Pune 411008, India \\ ${ }^{3}$ Academy of Science and Innovation Research (AcSIR), Ghaziabad, 201002, India. \\ *k.bhattacharjee@ncl.res.in; ${ }^{\text {pl.bhagavatula@ncl.res.in; }}$ \\ 91-20-25902013 (tel); 91-20-25902636 (Fax)
}

Section I: Materials and Experimental procedure:

Gold choride $\left(\mathrm{AuCl}_{3}, 99 \%\right)$, didodecyldimethylammonium bromide (DDAB, 98\%), 1dodacanethiol (DDT, 99\%) and sodium borohydride $\left(\mathrm{NaBH}_{4}\right)$ were purchased from Sigma Aldrich. Methanol, acetone, and toluene were procured from Thomas Bakar, India. All reagents were used without further purification. Extra pure Mili-Q water was used in all aqueous preparation.

Synthesis of Au NPs by DR method: For typical $0.01 \mathrm{M}$ Au NPs preparation, first $300 \mathrm{mg}$ DDAB and $90 \mathrm{mg}(0.01 \mathrm{~mole})$ of $\mathrm{AuCl}_{3}$ were dissolved in $30 \mathrm{~mL}$ toluene sequentially by sonication for $10 \mathrm{~min}$, in a $250 \mathrm{~mL}$ round bottled flask. A dark-orange solution appeared which was subsequently reduced by freshly prepared aq. $\mathrm{NaBH}_{4}$ solution $(250 \mu \mathrm{L} 9.4 \mathrm{M})$ by continuously stirring for $1 \mathrm{~h}$. After the reduction, to the resultant maroon-purple solution, $2.2 \mathrm{~mL}$ DDT (metal to DDT ratio 1:30) was added and stirred for another $15 \mathrm{~min}$ for ligand exchange to occur. Then the solution was precipitated with $150 \mathrm{~mL}$ methanol and the precipitate was collected at the bottom of the RB by gravitational sedimentation, followed by drying under flowing Argon. This dry mass was then dissolved in $30 \mathrm{~mL}$ toluene (same amount as in the beginning) under sonication and a second dose of DDT ( $2.2 \mathrm{~mL}, 1: 30$ ratio) was added. The resulting mixture was refluxed at $110{ }^{\circ} \mathrm{C}$ for $1 \mathrm{~h}$ under Argon atmosphere. After the reaction, the mixture was cooled down to room temperature and named as $A u D D T^{A P}$ sample. 


\section{Instrumentation and programming:}

Conventional bright field transmission electron microscopy (TEM) imaging was performed using Tecnai-G220S-Twin instrument with an acceleration voltage of $200 \mathrm{kV}$. Attenuated total internal reflection Fourier transformed infra-red spectroscopy (ATR-FTIR) was performed in the range of 2800 to $3200 \mathrm{~cm}^{-1}$ using Perkin Elmer, Spectrun-2 instrument. Thermogravimetric analysis was done in a Perkin Elmer, STA 6000 analyzer. Inductively coupled plasma-atomic emission spectroscopy (ICP-AES) analysis was done with an Agilent, 4200 spectrometer.

The simulation code was written in Python 3.5. Anaconda distribution for windows 64-bit operating system was used. The simulation was performed in a Dell work station, having $9^{\text {th }}$ generation i9-9900K processor, with 32 GB dual channel hyperx DDR4 RAM.

Fourier analyses and power spectral density calculation was performed using a modified Matlab code based on Ref 23.

Contact angle measurement: Surface wettability has an important role in required pattern formation upon solvent drying. Wetting is commonly characterized by the contact angle $(\theta)$, defined as, the angle between the tangent to the liquid-air interface and the solid surface at the three-phase contact line. ${ }^{9}$ In order to understand the evaporative mechanism of a sessile drop containing NP dispersion in the presence and absence of any free ligands, we performed contact angle measurements for different times during the evaporation process. The results were shown in figure SI-1). As soon as the fluid touches the substrate, the drop start spreading and reaches its maximum wetting diameter with contact angle of $\sim 32^{\circ}$. The solid-liquid-gas triple line were then pinned at that distance and evaporation is characterized by a decrease in contact angle and droplet height. After some time, a thin solvent film remains on the substrate which then evaporates slowly to dryness. From our experiment we have observed that presence of a nonevaporative (excess ligand in the present case) component within the drop can increase the timeline of the evaporation process (figure SI-1). One way to realize this is the change in saturated vapour pressure above the solution by selective solvent-ligand interaction. 


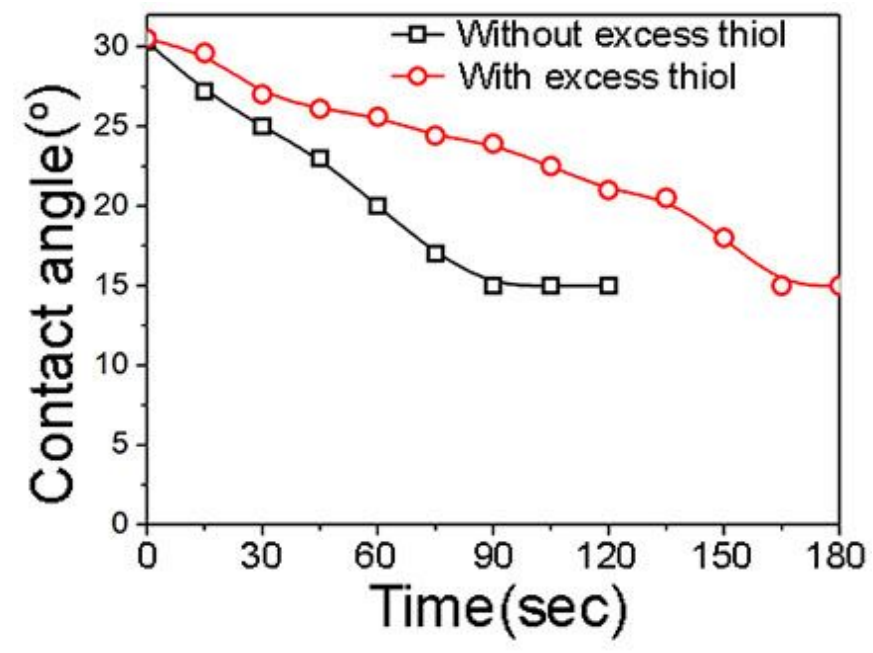

Figure SI-1: Plot of contact angle at different time of evaporation of a sessile drop.

Section II: Initial TEM screening

The initial particle concentration is an important factor to obtain 2D or 3D superlattices. For this we prepared three different samples which differ in particle concentration, by varying the dilution factor (DF) from the same $A u D D T^{A P}$ stock solution. It is done by, dispersing $10 \mu \mathrm{L}$ (DF100), $100 \mu \mathrm{L}$ (DF10), $250 \mu \mathrm{L}$ (DF4) of $A u D D T^{A P}$ in $1 \mathrm{~mL}, 0.9 \mathrm{~mL}$, and $0.75 \mathrm{~mL}$ of toluene, respectively. Then $5 \mu \mathrm{L}$ of each solution was drop casted in TEM grid as described in the text. Figure SII-1 shows the corresponding images for DF100, DF10, DF4 samples in the upper, middle and lower panel of the figure, respectively. Low magnification study reveals that large portion of NP monolayers were distributed throughout the TEM grid in case of DF100 sample (figure 1a)-c)). At high magnification we observed NPs are arranged in their close-to-equilibrium distribution which is a pseudo-hep structure within the monolayer, but occasionally filled with gaps, point defects, and twinning. In all other cases multilayer and/or 3D superlattices were found. Thus, we follow 100 times dilution sample as the $A u D D T^{A P}$ sample in the rest of the manuscript. 


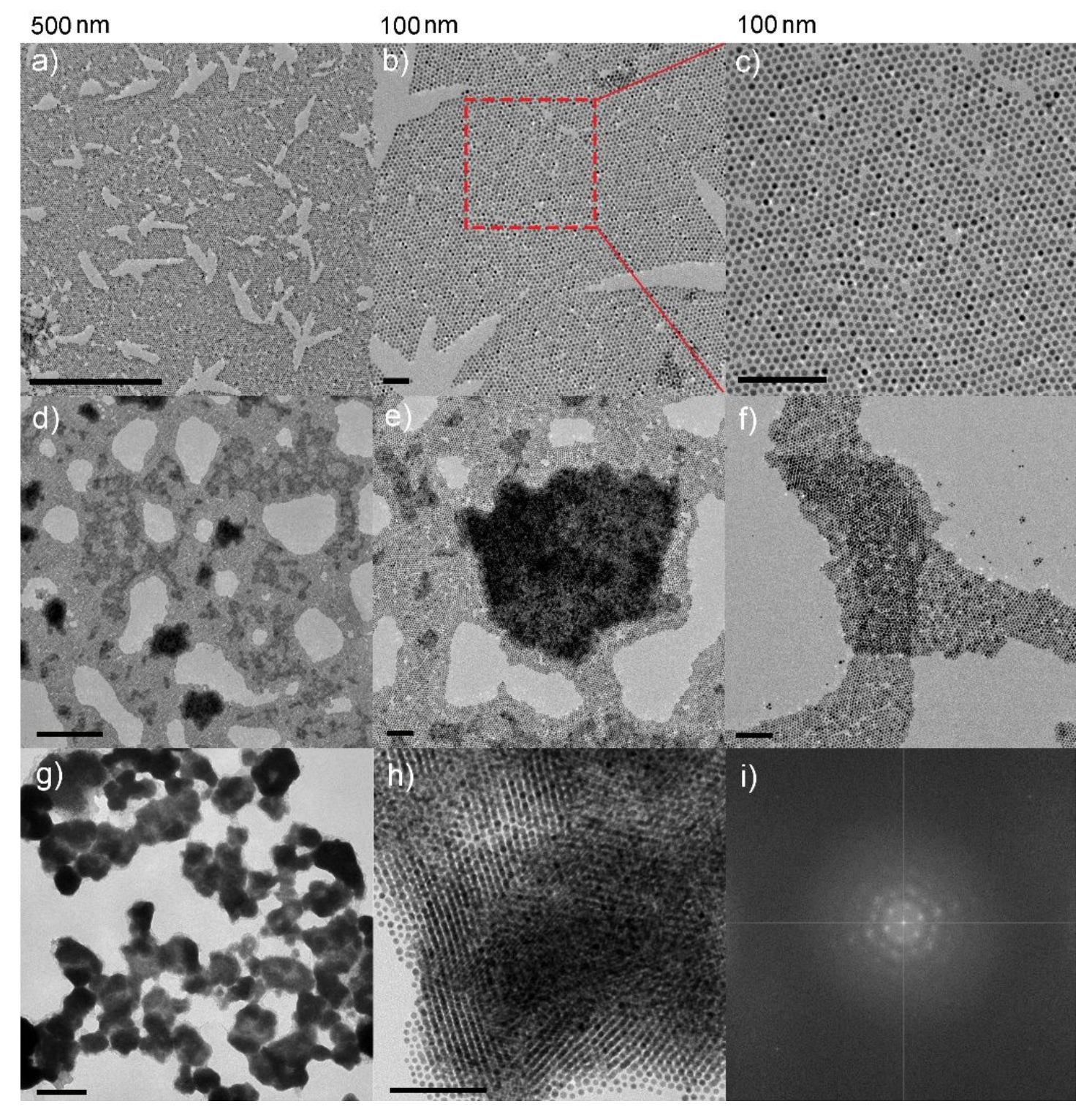

Figure SII-1: TEM images of a)-c) DF100; d)-f) DF10; g)-i) DF4 samples. Well distributed large-area monolayer was observed for the DF100 sample, upper panel. The darker spots in the middle layers for the DF10 sample are the bi/multi-layer super lattices. Large proportion of well crystalline 3D-superlattices were formed for the DF4 sample. FFT of figure h) was shown in figure i). Scale bar is shown on the top. 


\section{Section III: Image processing}

A detail about post-image processing by MIPAR ${ }^{\mathrm{TM}}$ can be found in the 'Mipar user manual' and their open source webinar. ${ }^{1}$ Here we noted all the background information that needed to support the method we used in the manuscript.

Creating recipe: A recipe is sequences of image processing steps, performed in a right order and with the right setting, in order to identify features of interest in an image. Once done, the whole sequence can be saved as a recipe file and can be applied later to a set of images for batch processing. Below is elaboration of some typical post processing tools we used in this study to create our required recipes.

Scale calibration: Allows us to calculate the pixel size in units of length rather than pixels.

Median filter: Helps to reduce electronic noise in the image. It set each pixel in the current image equal to the median pixel value of its neighbouring pixel with desired window size.

Non-local mean: Reduce image noise. Each pixel's local window is compared to window around it. The window's centre pixels are averaged together with weights depending on the variation between the windows.

Adjust contrast: Enhances image contrast and brightness by setting new black and white greyscale levels, and scaling the other levels accordingly.

Range threshold: Selects pixels if they are between a specified range of pixel values.

Adaptive threshold: This is a very effective tool while dealing with variation of pixel intensity at the edge. Using this one can selects pixels based on their intensity relative to some statistic of the surrounding neighbourhood. The typical values for two parameters, percentage (90\%) and window (70) mean if a pixel is less than $90 \%$ of the average grey scale value in a $70 \times 70$ pixels window around it, it will be selected.

Optimization of parameters by selection match: Perhaps the most critical yet less explored area of image processing is to quantify the accuracy of the image-processing algorithm in question. MIPAR $^{\mathrm{TM}}$ has provide with such facility where we can optimize parameter(s) to produce best similarity between the processed and original image. Here we adopted selection matching metric to choose the best parameter combination which yields a maximum selection match. In our 
recipe for NP counting we optimize the adaptive threshold parameter to accurately select the feature edge.

Reject feature: Reject desired features from the current image on the basis of feature's characteristics.

Fill all holes: Remove/fill holes within a feature if any.

Smart erosion: Smooth feature boundary using a specified window size. This is done by removing selected pixels if they are surrounded by a number of empty pixel greater than or equal to the specified number.

Separate feature: This is a very powerful tool which separates connected features using a watershed algorithm. A distance map of the image is made and interpreted as topography (i.e. basin within level ground). A rainfall simulation is then run such that connected features are separated where their basins fill to meet.

Smart cluster: In this process each pixel is assign to one of the specified classes using k-means clustering. ${ }^{2,3}$ This is a powerful and objective way of segmenting images. This function results in a grayscale image where each class is colored according to the specified statistic of the underlying pixels. To split the resulting image into different Layers, add a Set Memory image step afterwards, then iteratively call that restore image and use "Range Threshold" steps to select the different classes as $\mathrm{B} / \mathrm{W}$ images for global and/or feature measurements. This process involves three parameters: Fill type: select classes, which divide the image into number of classes and fills each cluster with value of its class. Image is then normalized from 0 to 255 for easier visualization. As we are trying here to segment one set of features from the background, we enter 2 classes. Edge clean: factor that controls extent to which edge artefacts should be removed. Speed: parameter that controls the resolution of the clusters. Lower speed results in higher resolution clustering.

Feature measurements: It allows us to measure a number of feature properties based on the features selected in the image. This includes, size parameters (area, caliper diameter, equivalent diameter, etc.), location parameters (centroid, nearest neighbour distance etc.), and shape parameters (eccentricity, aspect ratio, roundness, etc.) 
Global measurements: It allows us to perform one measurement per reconstructed image. This include, area fraction of feature (compared to the entire image), number density of feature, perimeter fraction (compared to the entire image).

Few important definitions used in the text:

Caliper diameter: Largest line length that fits across each feature.

Equivalent diameter: Diameter of each feature if each was a circle of the same area.

Nearest neighbour distance: Each feature's distance to the closest other feature, calculated from the features' centroids.

Roundness: Ratio of equivalent diameter to caliper diameter.

Area fraction: Count all the selected pixels in the reconstructed image and divides by the total number of pixels in the entire image.

Number density: Measures the total number of separate features per area in the current image.

Perimeter fraction: This is calculated as the total perimeter length divided by the total number of pixels in the image.

Recipe for NP counts: The recipe 1 for NP count is as follows,

1. Export the image

2. Median filter

3. Non-local mean filter

4. Adaptive threshold

This step has been optimized by "selection matching" algorithm which produces optimum combination of widow size and threshold value, simultaneously or separately.

5. Reject feature

6. Fill all holes

7. Smart erosion

8. Separate feature (Watershed algorithm, high separation and resolution)

9. Reject feature 
Figure SIII-1 shows the stepwise post-processing of a typical TEM image (for NP counting, figure 1b) in the manuscript) of AuDDT ${ }^{A P}$ sample. Figure SIII-2 shows the stepwise postprocessing of a typical TEM image (NP counting, figure $1 \mathrm{k}$ ) in the manuscript) of $A u D D T_{e x}^{0}$ sample

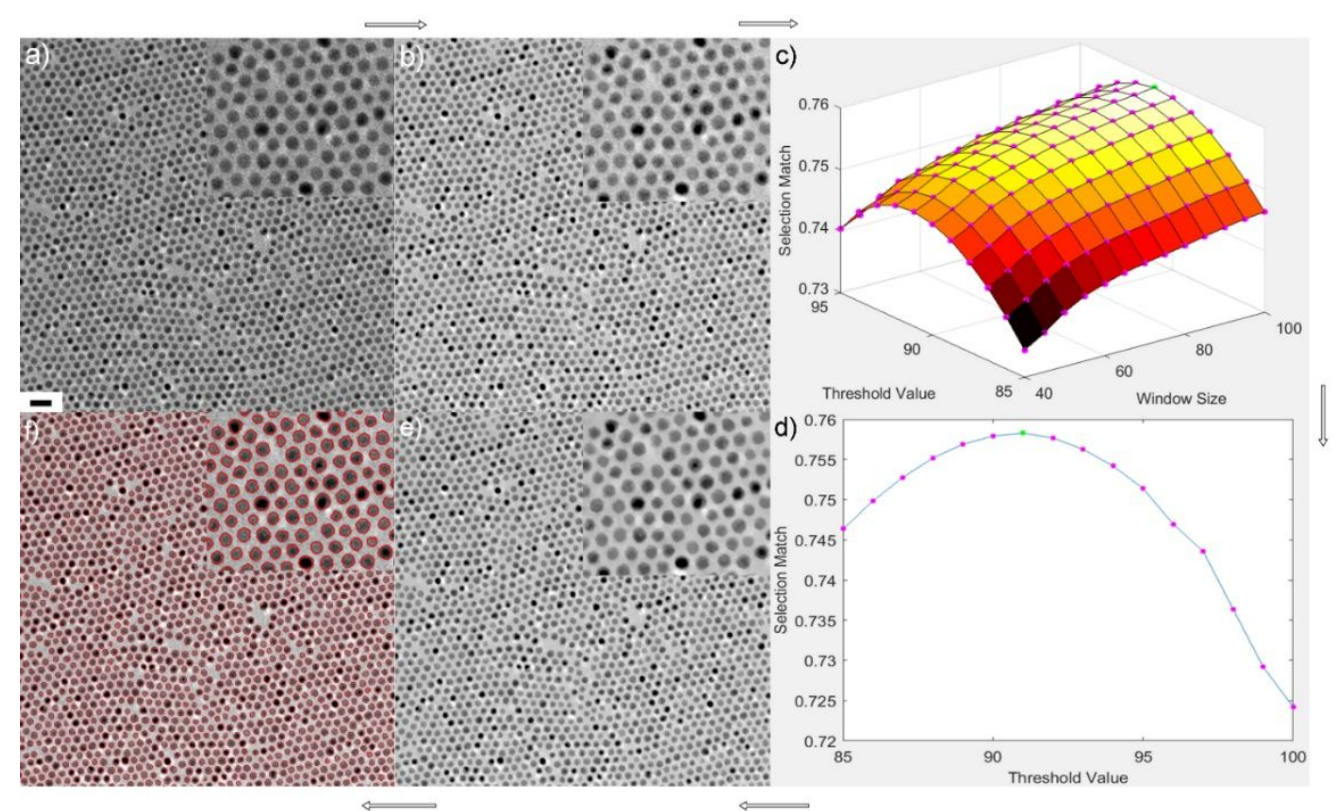

Figure SIII-1: Step-wise representation of typical image processing for NP mapping for $A u D D T^{A P}$ sample. a) original grey-scale, b) after non-local mean filter, c) and d) after two and single parametric optimization of adaptive threshold using selection match algorithm, respectively, e) after smart erosion, f) final optimized image with feature outline overlay. Insets show a small magnified portion from respective images. a), b), e), f) Scale bar is $20 \mathrm{~nm}$. 


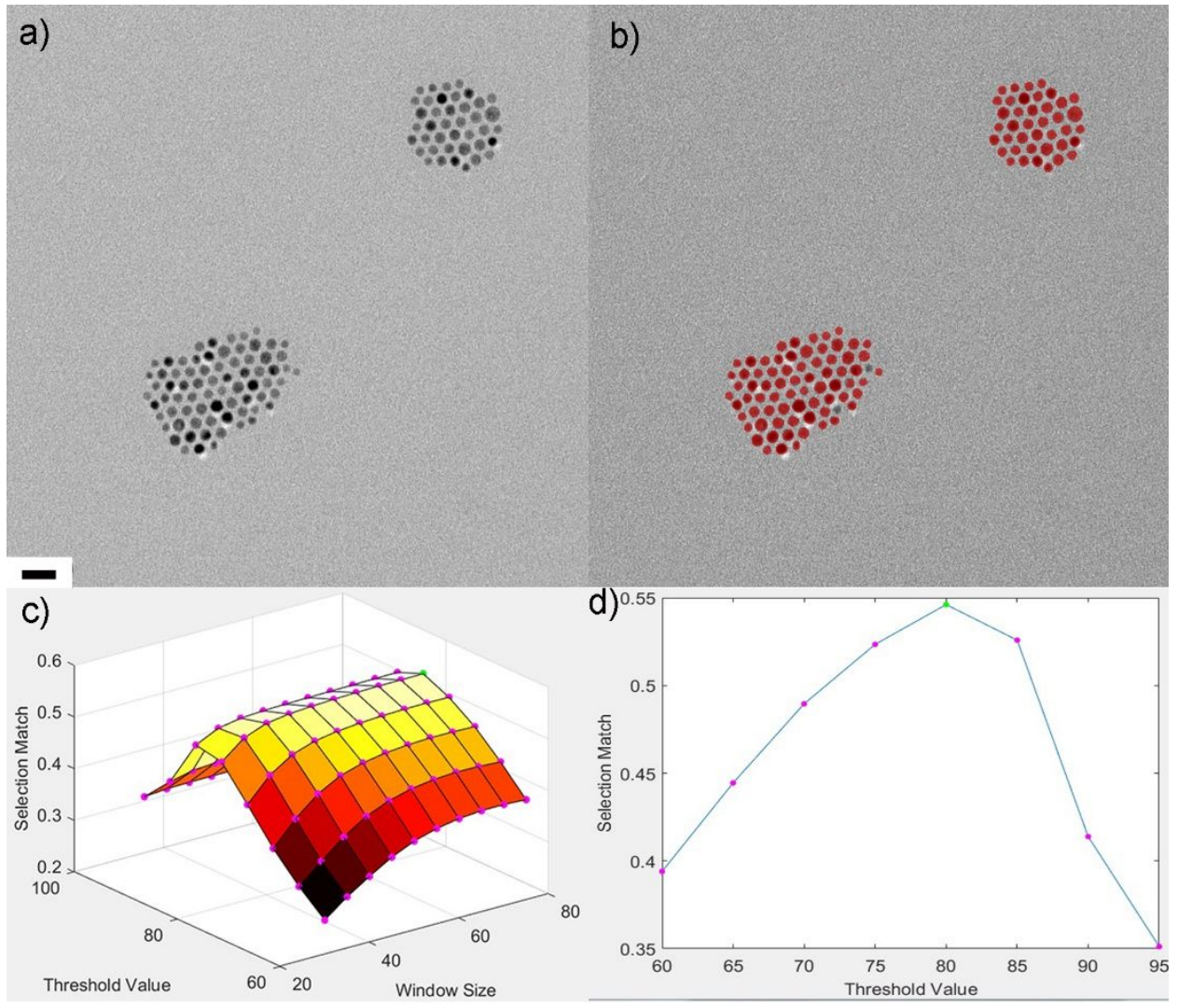

Figure SIII-2: Step-wise representation of typical image processing for NP mapping for $A u D D T_{e x}^{0}$ sample. a) original grey-scale, b) after thresholding, erosion and segmentation. c) and d) after two and single parametric optimization of adaptive threshold using selection match algorithm, respectively. a), b) Scale bar is $20 \mathrm{~nm}$

Recipe for pattern mapping: The recipe 2 for pattern mapping is as follows,

1. Export the image

2. Median filter (window size 5)

3. Non-local mean filter (window size 4 / strength 0.2)

4. Adjust contrast (auto)

5. Set memory image 1

6. Smart cluster (fill type: Class of 2; clean type: Dark to bright; edge clean: 1; speed: 2) Edit layer: Feature and Background

7. Call memory image 1

8. Range threshold (select feature) 


\section{Call memory image 1}

10. Range threshold (select background)

Figure SIII-3 shows the stepwise post-processing of a typical TEM image (for pattern mapping) $A u D D T_{e x}^{12}$ sample.

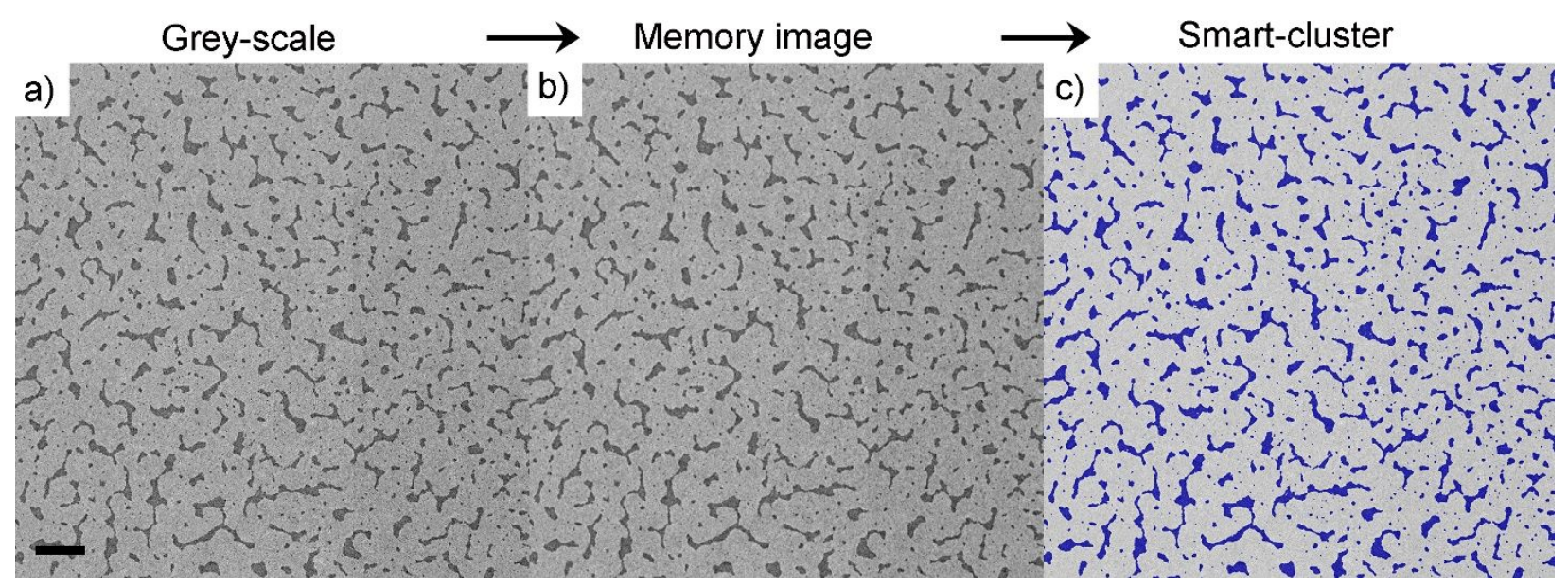

Figure SIII-3: Step-wise representation of typical image processing for mapping NP patterns using MIPAR. a) original greys-scale, b) Memory image 1, c) Smart cluster. a)-c) Scale bar 500 $\mathrm{nm}$.

Section IV: Supporting characterizations

ICP-AES analysis: In the present study we used the inductively coupled plasma atomic emission spectroscopy (ICP-AES) tool to measure the Au concentration and NP number density in the AuDDT sample. The detail calculation can be found in the Appendix I.

Sample for ICP-AES analysis were prepared as follows: $25 \mu \mathrm{L}$ of purified $A u D D T_{e x}^{0}$ sample was digested with $250 \mu \mathrm{L}$ of aqua-rigia $\left(\mathrm{HCL} / \mathrm{HNO}_{3}(1: 3 \mathrm{v} / \mathrm{v})\right)$ at room temperature for overnight in a sealed $5 \mathrm{~mL}$ glass vial. The solution was then diluted with $1 \mathrm{~mL}$ extra pure Mili-Q water. This solution was then subjected to ICP-AES for Au concentration analysis, after further diluting 10 times with water before measurement. Measurement performed with respect to standard gold solution in triplicate. A linear calibration curve was obtained, relating the count and gold concentration. The average gold atom concentration was measured to be $35 \mathrm{ppm}$ in the 
experimental solution. From this we calculated the gold atom concentration in the initial solution, which is found to be $0.009 \mathrm{M}$, close to our starting gold precursor concentration $(0.01$ M). Therefore, particle density in $A u D D T_{e x}^{0}$ stock solution was calculated from this concentration assuming a $2.7 \mathrm{~nm}$ radius of sphere as obtained from the TEM analysis is $10^{16}$ $/ \mathrm{mL}$. Detail calculation can be found in Appendix-I.

FTIR analysis: Figure SIV-1a) shows the comparison of the ATR-FTIR spectra of pure DDT molecule (in toluene) with respect to when grafted on gold NPs. The signal at $2570 \mathrm{~cm}^{-1}$ for the pure DDT molecule is responsible for the free S-H bond stretching vibration in the pure DDT molecule, which completely disappeared after get attached on the NP surface, signifying Au-S bond formation. A considerable effort has been devoted in the literature on the characterization of covalently attached alkanethiols by identifying their $\mathrm{C}-\mathrm{H}$ stretching vibrations in the wavenumber range 2800 to $3000 \mathrm{~cm}^{-1} .^{5}$ In our case, we observed the narrowing and sharpening of peak width when the molecules get attached to the gold surface. This is probably due to the partial loss of ligand's degrees of freedom upon bonding through one end. It also supports the fully extended configuration of ligand with fewer gauche defects. We deconvoluted both the spectrum and found four characteristic frequencies which are shown in the right side of figure SIV-1a). Vibrations at $2852 \mathrm{~cm}^{-1}$ and $2940 \mathrm{~cm}^{-1}$ were due to the symmetric and asymmetric stretching vibration of $\mathrm{CH}_{2}$ group, respectively. While, the signals at the wavenumber $2880 \mathrm{~cm}^{-1}$ and $2967 \mathrm{~cm}^{-1}$ were due to the symmetric and asymmetric vibration of the terminal $\mathrm{CH}_{3}$ group. The reduction in the ratio of the area under the curves for symmetric and asymmetric stretching upon surface grafting clearly indicates our previous conjecture.

Thermogravimetric analysis: There are varieties of analytical techniques discussed in the literature for the characterization of ligands on the nanoparticle surface and for the calculation of ligand density associated with it. ${ }^{6,7,8}$ Among them, thermogravimetric analysis (TGA) is the most acceptable technique to measure the residual mass after heating a dried sample at high temperature. For gold NPs covered with n-alkyl ligands, the residual mass, is due to the inorganic core. Figure SIV-1b) shows the weight-temperature characteristics for the dried $A u$ $D D T_{e x}^{0}$ powder sample. The carbon content determined by this technique validated the solution phase ligand grafting density. The detail calculation procedure is given in the Appendix I. 
Sample for TGA were prepared as follows: $5 \mathrm{~mL}$ of purified $A u D D T_{e x}^{0}$ sample was further precipitated and dried in a vacuum oven for overnight at $80{ }^{\circ} \mathrm{C}$. As-collected dried powder sample ( 12 mg) was then subjected to TGA, where, it is first heated up to $100{ }^{\circ} \mathrm{C}$ in air at a heating rate $5{ }^{\circ} \mathrm{C} / \mathrm{min}$ and hold there for $30 \mathrm{~min}$ to remove trace amount of organic content and moisture if any, followed by, heating at $5{ }^{\circ} \mathrm{C} / \mathrm{min}$ rate up to $500{ }^{\circ} \mathrm{C}$. The percentage mass loss was then used to calculate the ligand grafting density.

a)
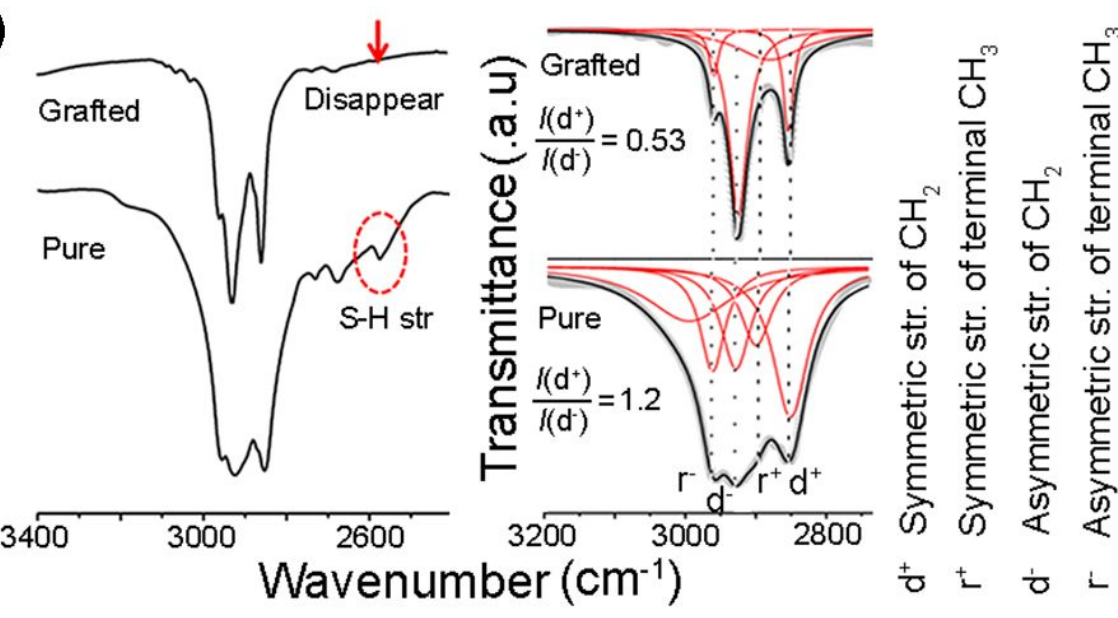

b)

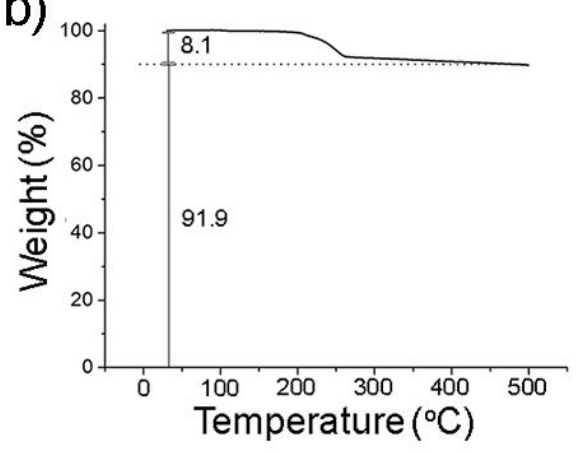

Figure SIV-1: a) Comparison of ATR-FTIR spectra of pure DDT and DDT-coated Au NPs solution in the $\mathrm{CH}_{2}$ stretching vibration region and their decomposition. b) TGA curve of the DDT-coated Au NPs powder sample indicating relative proportion of metal (91.9\%) to ligand $(8.1 \%)$. 
Section V: Supporting TEM analysis

In this section we summarize selective TEM images for all the samples discussed in the manuscript taken at different magnification. In the upper panel of Figure SV-1 shows the results. In the lower panel we described the domain mapping analysis using a local FFT correlation to selected references built-in with Mipar. Two different domains are first selected as reference then pattern analysis was done using a window size greater than twice of the NP size. This create a mapping of different domain according to which 'reference; is matches the best, as indicated in red and blue. From this analysis we confirmed that different domains can merge with each other with different orientation as a result of non-equilibrium driving force.

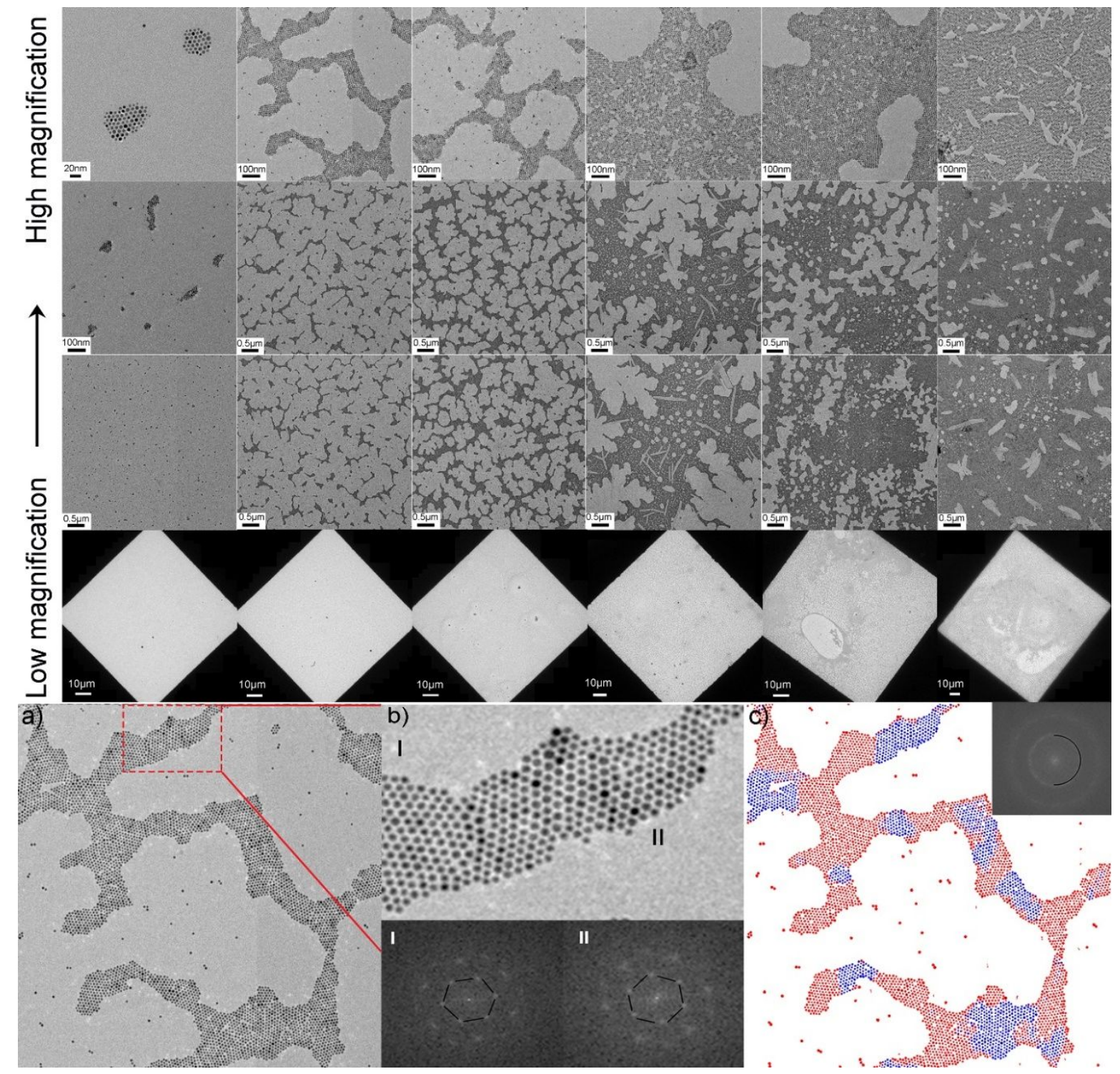


Figure SV-1: Upper panel: TEM images at different magnification for all the $A u D D T_{\text {ex }}$ samples mentioned in the text. Left to right excess ligand concentration increases. Lower panel: a) TEM image of $A u D D T_{\text {ex }}^{12}$ sample. b) Magnified image of the selected area as shown by the square box. FFT of two different areas as shown in the image was given below. c) Different domains in the original image are mapped using FFT correlation algorithm. Red and blue colour represents domain I and II. The FFT of the entire image is shown in the inset.

\section{Section VI: Monte Carlo Model}

Lattice-gas approximation: In our model, the simulation box is partitioned into imaginary microscopic square unit called 'cell' of side length equal to the correlation length of solvent $(\xi \approx$ $1 \mathrm{~nm}$ ). We define a square lattice (see figure SVI-1) where a single lattice cell $i$, can occupy by one of these three binary variables solvent $\left\{s_{\mathrm{i}}=1 ; n_{\mathrm{i}}, 1_{\mathrm{i}}=0\right\}$, nanoparticle $\left\{n_{\mathrm{i}}=1, s_{\mathrm{i}}, l_{\mathrm{i}}=0\right\}$, or ligand $\left\{l_{\mathrm{i}}=1 ; n_{\mathrm{i}}, s_{\mathrm{i}}=0\right\}$. A single lattice site cannot be occupied by more than one species to incorporated excluded volume effect. Periodic boundary conditions apply to the lattice. Latter we will assume that number of species is either fixed (as in case of NP and ligand) or can fluctuate (as in case of solvent) during evaporation. These two descriptions are called canonical and grand-canonical, respectively; together with produce a semi grand-canonical description in our system. ${ }^{10}$ As it was previously pointed out that the size of the NP is not crucial, ${ }^{11,12}$ therefore, in our implementation, the solvent and nanoparticle occupy single cell in the lattice. A schematic representation of this model is shown in figure SVI-1. The interaction between two species of similar kinds, viz, solvent-solvent, NP-NP, and ligand-ligand were designated as $\varepsilon_{s s}$, $\varepsilon_{n n}$, and $\varepsilon_{l l}$, respectively. While the interaction between two unlike species, such as, nanoparticle-solvent, ligand-solvent, and nanoparticle-ligand were termed as $\varepsilon_{n s}, \varepsilon_{l s}$, and $\varepsilon_{n l}$, respectively. For simplification, substrate interaction was neglected altogether. The Hamiltonian used in our simulation is formulated as follows,

$$
H=-\varepsilon_{s s} \sum_{<i j>} s_{i} s_{j}-\varepsilon_{n n} \sum_{<i j>} n_{i} n_{j}-\varepsilon_{l l} \sum_{<i j>} l_{i} l_{j}-\varepsilon_{n s} \sum_{<i j>} n_{i} s_{j}-\varepsilon_{l s} \sum_{<i j>} l_{i} s_{j}-\varepsilon_{n l} \sum_{<i j>} n_{i} l_{j}-\mu(v) \sum_{i} s_{i}
$$

where, $\langle i j\rangle$ indicates summation over pair of nearest neighbour interactions and $\mu(v)$ is the effective chemical potential as described below. 


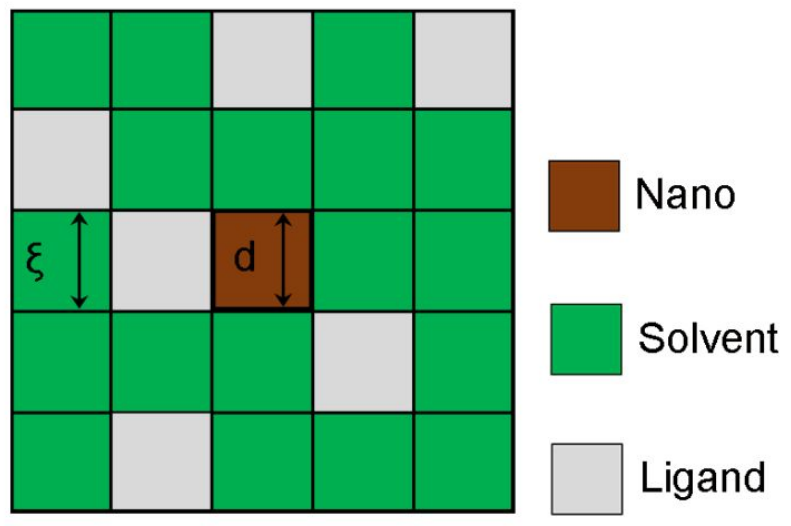

Figure SVI-1: Sketch of the 2D square lattice constructed for our MC model. In our code the length of NP (d) span one unit-cell similar to the solvent correlation length $(\xi)$.

Parameter space for the simulation: The value of $\varepsilon_{s s}$ was set -0.5 and all other interactions are scaled with respect to this value. Unless stated otherwise, we choose, $\varepsilon_{n n}=2 \varepsilon_{s s}=-1, \varepsilon_{l l}=\varepsilon_{s s}$ $=-0.5, \varepsilon_{n s}=1.5 \varepsilon_{s s}=-0.75, \varepsilon_{l s}=1.5 \varepsilon_{s s}=-0.75, \varepsilon_{n l}=0, \mathrm{NMRF}=4, \mathrm{LMRF}=4, \mathrm{EVRF}=$ 0.04 , throughout the simulation. Few silent features of these assumptions are, 1) $\varepsilon_{n n}>\varepsilon_{n s}$ in magnitude, means NPs strongly attract (the magnitude of the value) each other than solvent or ligand which implies the core is more polarizable. ${ }^{11} 2$ ) in values, $\varepsilon_{s s}>\varepsilon_{n s}>\varepsilon_{n n}$, which mean that the interaction between NPs is effectively screened in solution preventing it from aggregation, and manifested only when solvent evaporates. $\left.{ }^{11,12} 3\right) \varepsilon_{n s}>\varepsilon_{n n}$ which implies that NPs have good solubility in the solvent. 4) $\varepsilon_{l s}>\varepsilon_{l l}$ in magnitude, means ligands have good solubility in the solvent. 5) $\varepsilon_{n l}<\varepsilon_{l l}$ in magnitude, means ligands have repulsive interaction with NPs.

In the grand canonical ensemble, the system can exchange material with an external reservoir of fixed chemical potential, $\mu$. The value of chemical potential and the temperature concurrently determine the evaporation (and condensation) process. As the solvent density fluctuates during evaporation, we make the chemical potential an explicit function of global liquid solvent coverage $(v)$ by expressing it in the form $\mu(v)=v_{0}+\left(B \mu_{0} \times v^{A}\right),{ }^{13}$ where $v$ is the fraction of solvent that has become vapour and $\mu_{0}$ is the starting value of $\mu$ for the simulation, $\mathrm{A}$ and $\mathrm{B}$ are two parameters fixed at the value of 0.5 and 0.7 , respectively. We performed the simulation in 
the early spinodal limit ${ }^{12}$ where, $\mu_{0}$ is selected as, $\mu_{0}=2 \epsilon_{s s}-2 \varphi_{n}\left(\varepsilon_{n l}+\varepsilon_{s l}-\varepsilon_{s s}\right)$ and the temperature is near the critical temperature $\sim 0.57 \varepsilon_{s s} . \varphi_{n}$ is the NP area fraction, calculated from the TEM analysis (figure 2g) in the manuscript) NPs are allowed to move both in the direction of solvent and ligand filled area, but not in the direction of vapour.

\section{Monte Carlo moves:}

Three stochastic moves, nanoparticle diffusion, ligand diffusion and solvent evaporation/condensation were considered in a sequential order. These moves are always accepted with a Metropolis acceptance probability, ${ }^{14} P_{a c c}=\min \left\{1, \exp \left(\frac{-\Delta H}{K_{B} T}\right)\right\}$, where $\Delta H$ is the change in energy associated with the move, $T$ is the temperature of the heat bath, $\mathrm{k}_{\mathrm{B}}$ is the Boltzmann's constant. Three consecutive cycles were performed in one complete MC step: nano cycle, ligand cycle, and solvent cycle.

Nano cycle: Diffusion of NP was done first, the following steps are repeated $N M R F \times \mathrm{n}$ times, where $\mathrm{n}$ is the number of NPs and $N M R F$ is the nanoparticle mobility ratio factor as defined below. i) Select a random NP from the lattice, ii) chose a direction into which it will move with probability 0.25 (up, down, left, right), iii) if three cells in the selected direction adjacent to the $\mathrm{NP}$ are either filled with liquid solvent or ligand or both, determine $\Delta \mathrm{H}$ from equation 1 , if not, this trial move is rejected, iv) if the previous step is successful, accept the move using Metropolis acceptance probability according to equation 2 . Liquid solvent and ligand cell are not allowed to interchange in this cycle. Ligand cycle: Diffusion of ligand was then performed by four steps similar to above and repeated $L M R F \times \mathrm{n}$ times, where, $\mathrm{n}$ is the number of ligand and $L M R F$ is the ligand mobility ratio factor as defined below. As the ligand correlation length is in the same order of solvent, ligands are allowed to move in the direction of liquid solvent only. Solvent cycle: Solvent phase change was considered next. Two separate cycles for solvent evaporation (convert liquid to vapour) and condensation (convert vapour to liquid) were carried out specific times related to the evaporation ratio factor and condensation ratio factor, respectively. The typical cycle has following steps. i) Select a random solvent from the lattice, ii) if the selected solvent cell is liquid then convert it to vapour (or if vapour convert it to liquid) and determine $\Delta \mathrm{H}$ from equation 1 for the process, iii) If the previous step was successful, the move was accepted using Metropolis acceptance probability. This MC step was then repeated several times 
until all liquid evaporates leaving the characteristic pattern resulted from NP self-assembly. A flow chart diagram of the algorithm discussed above was shown in the figure SVI-2.

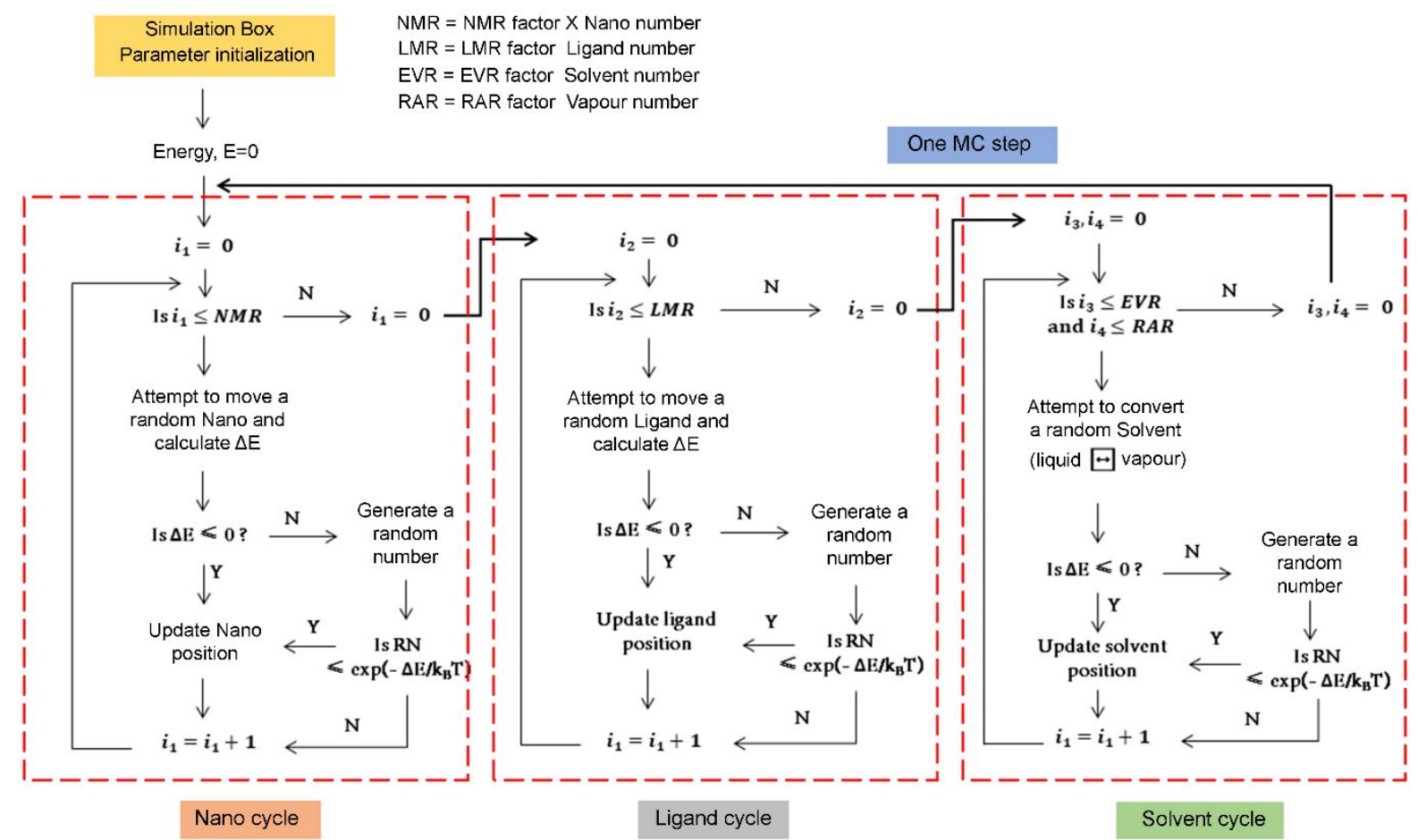

Figure SVI-2: Flow-chart of our MC algorithm showing the individual cycles associated within.

Diffusion coefficient and time scaling: When we referred the system is evolved for a time of $x$ Monte Carlo steps (MC steps), that means on average there has been at least $x$ times per lattice site attempt made to move on. The physical time scale is related to the MC steps by diffusion coefficient (D). ${ }^{11}$ For a single NP moving through bulk liquid, D can be calculated by measuring the distance $(r)$ that it travels at a certain time interval $(t)$ and repeat that measurement for a sufficiently long simulation time. It was assumed that the NP has zero interaction with the environment during the movement and evaporation was ignored. The time average mean square displacement (TA-MSD), was calculated as ${ }^{15}$

$$
<\Delta^{2} r(t)>=\frac{1}{N} \sum_{i=1}^{N}<\left[R_{i}(t)-R_{i}(t)\right]^{2}
$$


where, $\mathrm{R}(t)$ is the position of $i^{\text {th }} \mathrm{NP}$ in the liquid. The quantity inside the angular brackets is the square of the displacement that a NP has undergone during time interval $t$. Summing over all the NP and dividing by the number of NP gives the mean value. The connection between MSD and the diffusion lies in the dependency of $\left\langle\Delta^{2} r(t)\right\rangle$ at long times. Figure SVI-3 shows that for NP at time long compared to typical molecular level time, $\left\langle\Delta^{2} r(t)\right\rangle$ varies linearly with time, which is a characteristic of diffusive motion. In this case the slope of $\left\langle\Delta^{2} r(t)\right\rangle$ is proportional to the diffusion coefficient, $D ;\left\langle\Delta^{2} r(t)\right\rangle=4 D t .{ }^{11}$ Change in MSD for different NMRF values shows a linear dependence, therefore, MSD is chosen as the representative of diffusion constant. We use $\mathrm{NMRF}=4$, for all the calculation. $\mathrm{D}$ is calculated to be, $D=0.2 \sigma^{2} / \mathrm{MC}$ step, where $\sigma$ is the diameter of the NP taken as unity in the present case. The Brownian timescale $\left(\tau_{B}\right)$ is related to the diffusion coefficient by, ${ }^{11} \tau_{B} \equiv \sigma^{2} / 4 D \approx 1.25 \mathrm{MC}$ steps. $\tau_{B}$, is the average time taken by a $\mathrm{NP}$ to diffuse a distance equal to its own diameter. We specify time in the unit of MC steps in the text.

Definition of important terms used in the text:

Nano Mobility Ratio Factor (NMRF): Average number of attempted displacement (in the unit of one smallest box of the simulation area) of NP in one nanocycle.

Ligand Mobility Ratio Factor (LMRF): Average number of attempted displacement (in the unit of one smallest box of the simulation area) of ligand in one ligand cycle.

Evaporation Ratio factor (EVRF): Average number of attempted deletion (in the unit of one smallest box of the simulation area) of solvent cell (liquid $\rightarrow$ vapour) in one solvent cycle.

Reappearance Ratio Factor (RARF): Average number of attempted reappearance (in the unit of one smallest box of the simulation area) of solvent cell (vapour $\rightarrow$ liquid) in one solvent cycle.

Mean Square Displacement (MSD): Number average of the square of the displacements of nanoparticles after $n$ MC steps ( $n$ is a large number) starting from the origin under nonevaporative and non-interactive condition.

Cluster Size (CS): An average number of nearest neighbour NPs around a central NP. 


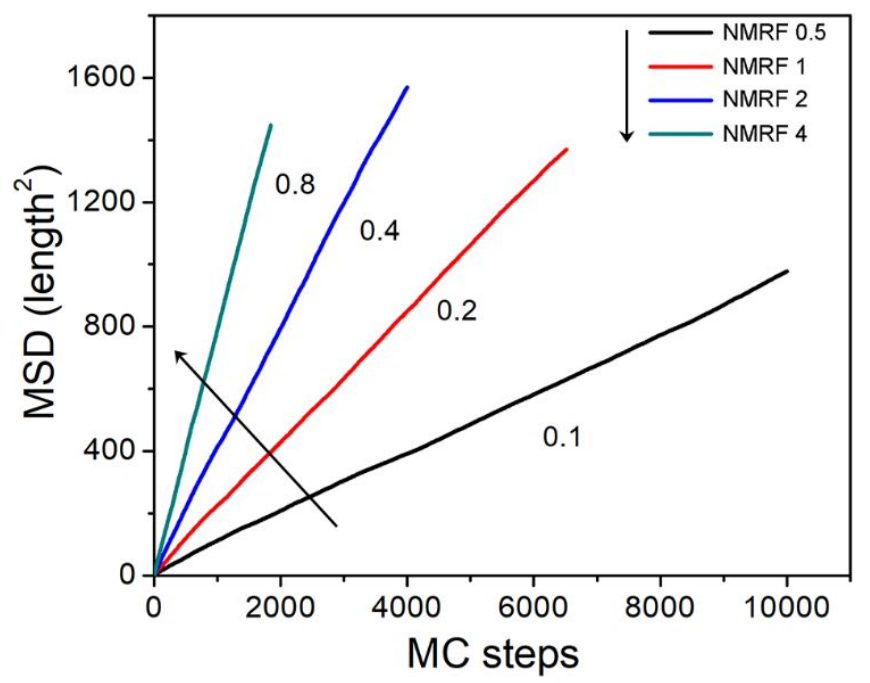

Figure SVI-3: Number average mean square displacement (MSD) vs. simulation time plot for different NMR values. Diffusion coefficient was calculated from the slope of the curves. Simulation was performed for box size $77 \times 77, \varphi_{n}=0.2, \varphi_{l}=0$, all interactions are set to $0, \mathrm{kT}=$ $0.3, \mu=0.8,10^{5} \mathrm{MC}$ steps have been performed. Arrows are for guide to the eye.

Parameter optimization: Two newly introduced interaction parameters, viz, $\varepsilon_{n l}$ and $\varepsilon_{l s}$, were optimized by varying each of them at a time, while keeping other parameters fixed at values as specified in the caption of the Figure SVI-4, SVI-5. We observed, the time (MC steps) required for compete solvent evaporation increases with increase in $\epsilon_{l s}$ and decrease in $\epsilon_{n s}$. In addition, at their optimum values, the system gradually evolves toward complete spinodal decomposition and concomitantly increases in cluster size. From this analysis, the optimum values for $\varepsilon_{l s}$ and $\varepsilon_{n l}$ are set to be -0.75 and 0 , respectively. Next, we studied the effect of NMRF, LMRF, and EVRF on the final NP pattern formation keeping other parameters at their values as mentioned in the caption of the corresponding Figure caption.

We observed a nominal improvement in the final pattern while NMRF in the range, $1<$ NMRF $<$ 4 (see Figure SVI-6). So, we fixed the optimum value for NMRF $=4$. We found no such dependency of LMRF on the final pattern formation, and therefore it is fixed at LMRF $=1$. We noticed a significant influence of EVRF on the final NP pattern (see Figure SVI-7). EVRF is defined as the average number of attempted deletion (in the unit of one smallest box of the simulation area) of a solvent cell (liquid $\rightarrow$ vapor) in one solvent cycle. We find that with the 
increase in EVRF (i.e. with a greater number of solvent deletions in a single MC step) the nature of the pattern changes from large interconnected domains to small isolated islands. We attribute this to the fact that the effective number of solvent molecules disappear faster than the nanoparticles diffusing through it. Nano fraction, $\varphi_{n}$ were also scrutinized from $0.1 \leq \varphi_{n} \leq 0.5$ (see Figure SVI-8). The patterns change dramatically with increase in nano fraction, from isolated island, to worm-like arrangement, to interconnected domains.
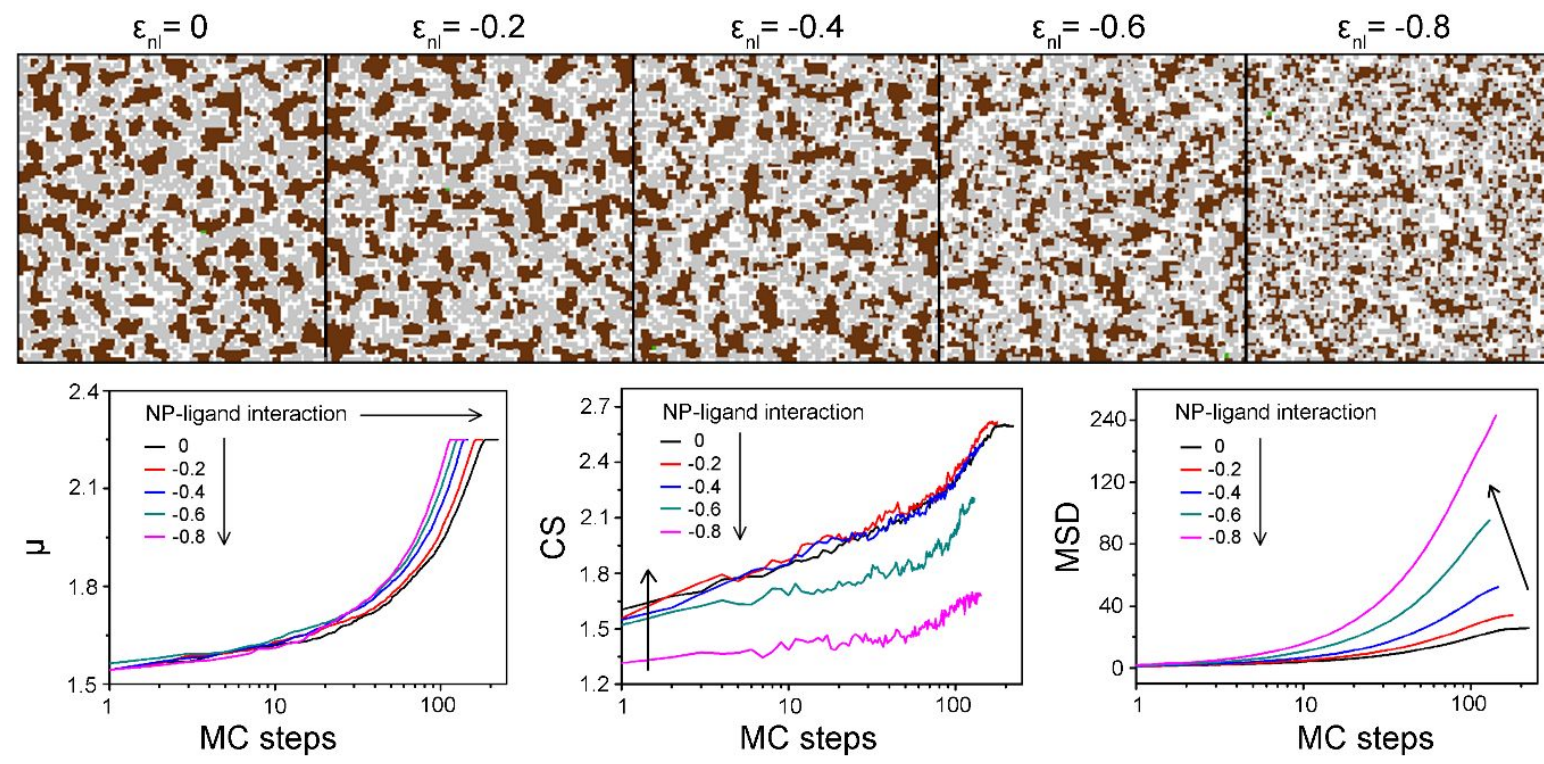

Figure SVI-4: Final simulated pattern for different solvent-ligand $\left(\varepsilon_{\mid s}\right)$ interaction values as mentioned at the top of the images. Other fixed parameters are, box size $=77 \times 77, \varphi_{1}=0.3, \varphi_{\mathrm{n}}$ $=0.3, \varepsilon_{\mathrm{ss}}=\varepsilon_{l l}=-0.5, \varepsilon_{\mathrm{nn}}=-1, \varepsilon_{\mathrm{ns}}=-0.75, \varepsilon_{\mathrm{nl}}=0, \mathrm{NMRF}=4, \mathrm{LMRF}=1, \mathrm{EVRF}=0.04$, $\mu_{0}=1.5, \mathrm{kT}=0.3$. Below are the plots for chemical potential, cluster size and mean square displacement with simulation time for different ligand-solvent interaction values. Arrows are for guide to the eye. 

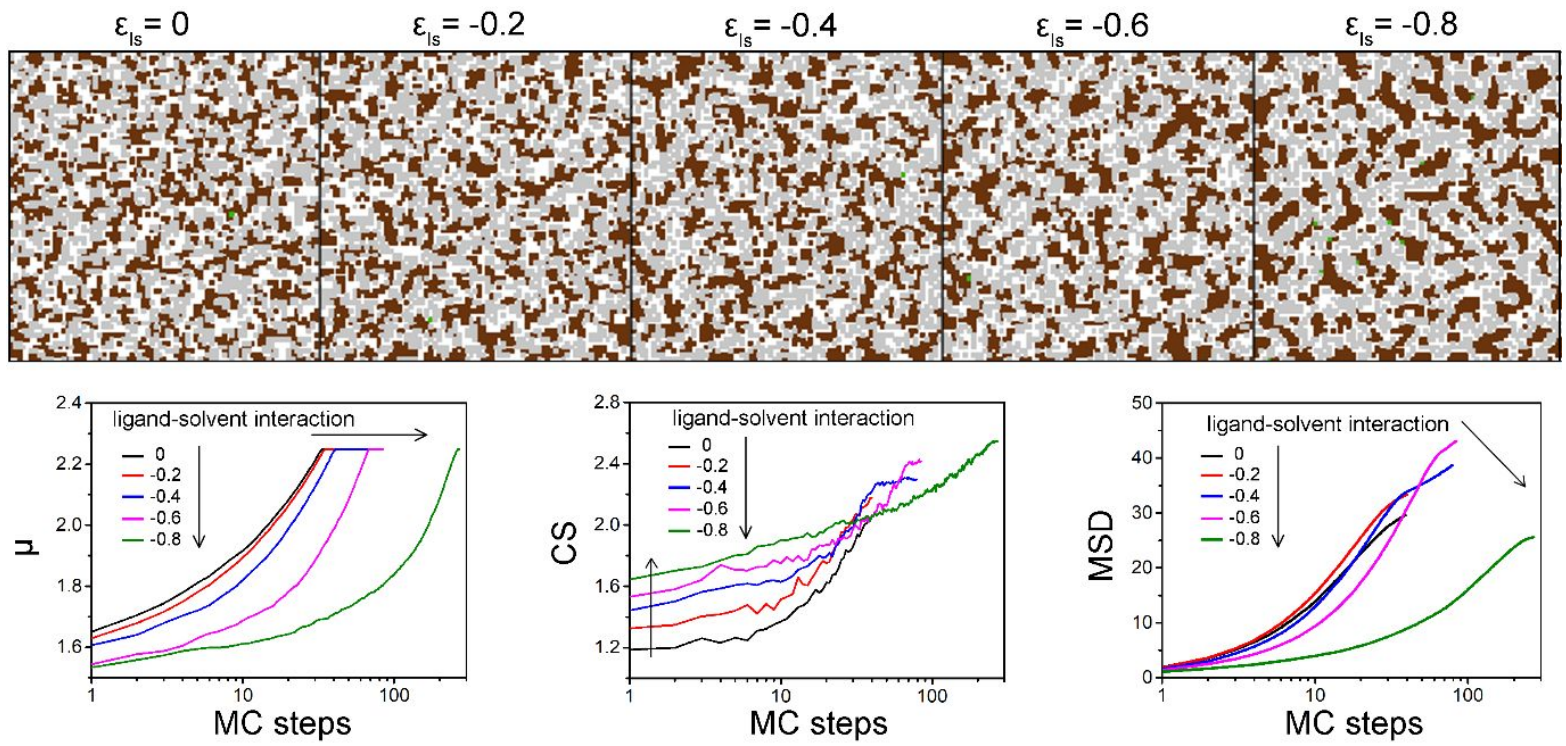

Figure SVI-5: Final simulated patterns for different solvent-ligand $\left(\varepsilon_{\mathrm{nl}}\right)$ interaction values as mentioned at the top of the images. Other fixed parameters are, box size $=77 \times 77, \varphi_{\mathrm{l}}=0.3, \varphi_{\mathrm{n}}$ $=0.3, \varepsilon_{\mathrm{ss}}=\varepsilon_{\mathrm{ll}}=-0.5, \varepsilon_{\mathrm{nn}}=-1, \varepsilon_{\mathrm{ns}}=-0.75, \varepsilon_{\mathrm{ls}}=-0.75, \mathrm{NMRF}=4, \mathrm{LMRF}=1, \mathrm{EVRF}=$ $0.04, \mu_{0}=1.5, \mathrm{kT}=0.3$. Below are the plots for chemical potential, cluster size and mean square displacement with simulation time for different NP-ligand interaction values. Arrows are for guide to the eye. 

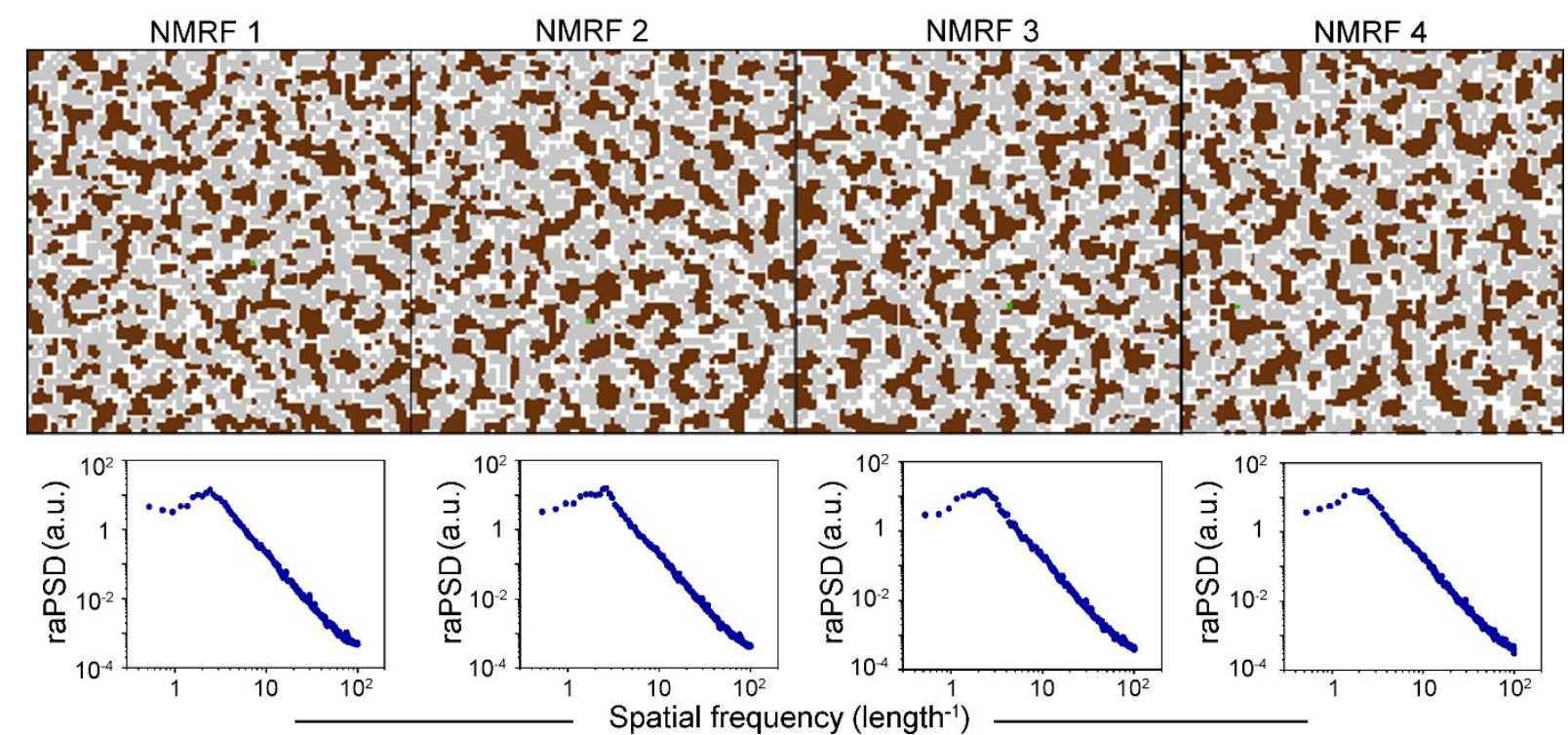

Figure SVI-6: Final simulated pattern for different nano mobility ratio factor (NMRF) as mentioned at the top of the images. Other fixed parameters are, box size $=77 \times 77, \varphi_{1}=0.3, \varphi_{\mathrm{n}}=$ $0.3, \varepsilon_{\mathrm{ss}}=\varepsilon_{l l}=-0.5, \varepsilon_{\mathrm{nn}}=-1, \varepsilon_{\mathrm{ns}}=-0.75, \varepsilon_{\mathrm{nl}}=0, \varepsilon_{\mathrm{ls}}=-0.75, \mathrm{LMRF}=1, \mu_{0}=1.5, \mathrm{kT}=$ 0.3. Corresponding radial average of power spectral density plot was shown at the bottom of each image.

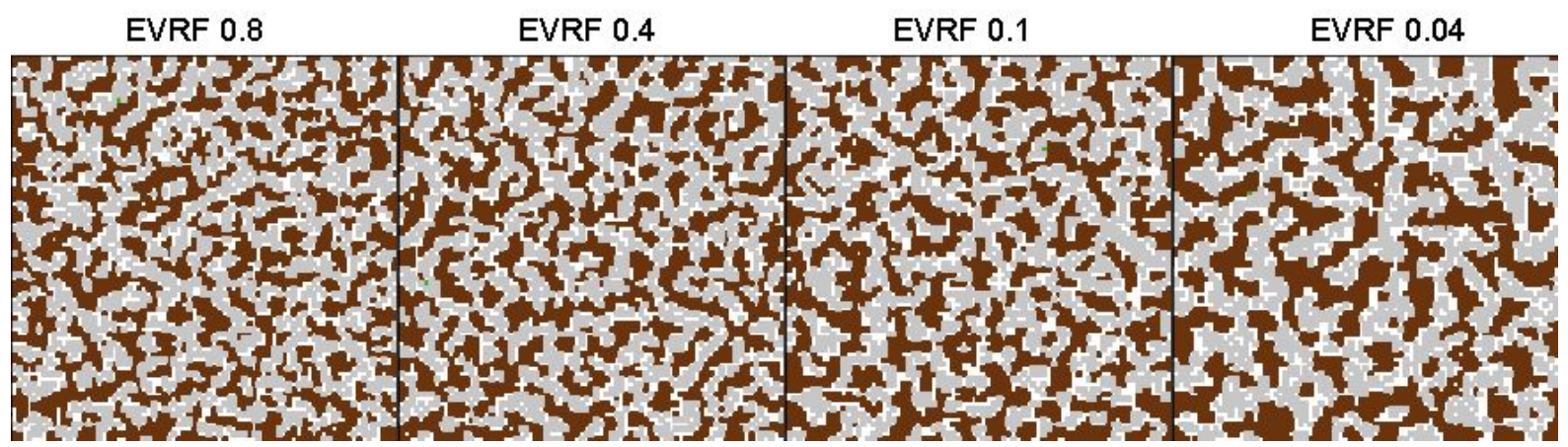

Figure SVI-7: Final simulated pattern for different evaporation ratio factor (EVRF) as mentioned at the top of the images. Left to right evaporation rate decreases. Other fixed parameters are, box size $=77 \times 77, \varphi_{\mathrm{l}}=0.3, \varphi_{\mathrm{n}}=0.3, \varepsilon_{\mathrm{ss}}=\varepsilon_{\mathrm{ll}}=-0.5, \varepsilon_{\mathrm{nn}}=-1, \varepsilon_{\mathrm{ns}}=-0.75$, $\varepsilon_{\mathrm{ls}}=-0.75, \varepsilon_{\mathrm{nl}}=0, \mathrm{NMRF}=4, \mathrm{LMRF}=1, \mu_{0}=1.5, \mathrm{kT}=0.3$. 


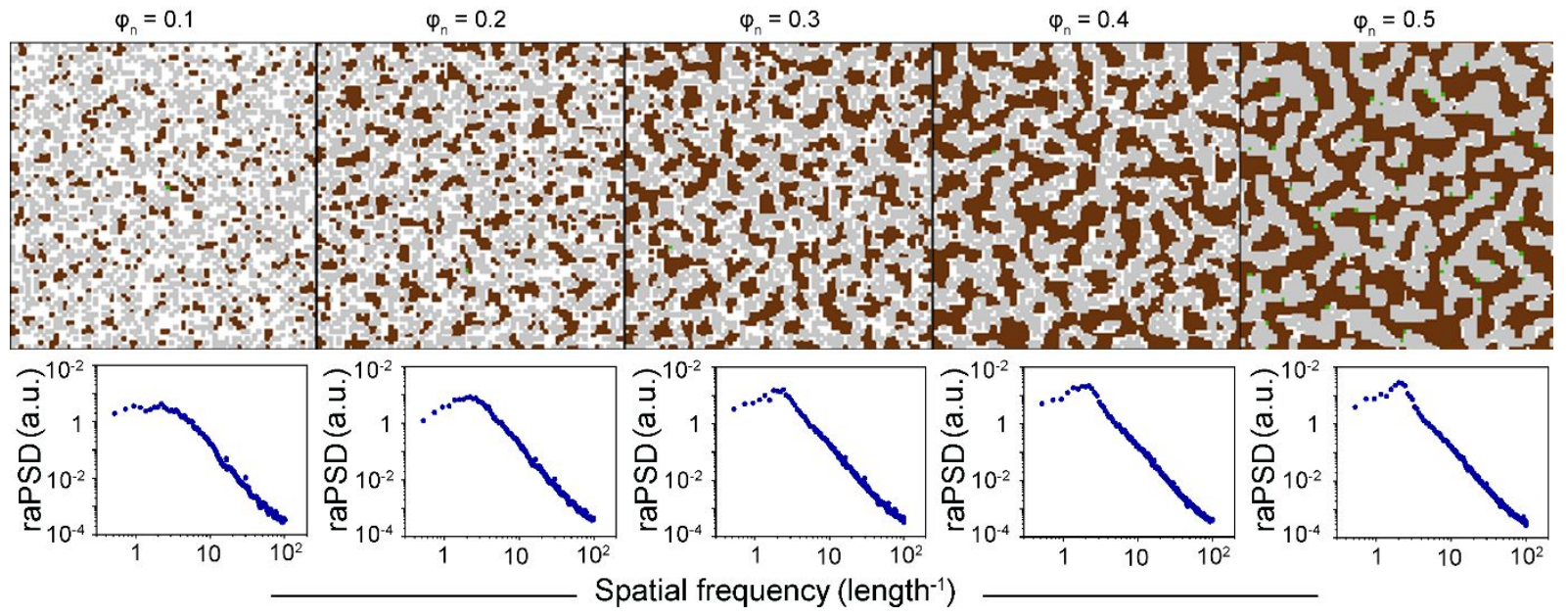

Figure SVI-8: Final simulated pattern for different nano fractions $\left(\varphi_{n}\right)$ as mentioned in the images in presence of excess ligand $\left(\varphi_{\mathrm{l}}=0.3\right)$. From left to right nano fraction increases. Other fixed parameters are, box size $=77 \times 77, \varphi_{\mathrm{l}}=0.3, \varepsilon_{\mathrm{ss}}=\varepsilon_{\mathrm{ll}}=-0.5, \varepsilon_{\mathrm{nn}}=-1, \varepsilon_{\mathrm{ns}}=-0.75, \varepsilon_{\mathrm{nl}}$ $=0, \varepsilon_{\mathrm{ls}}=-0.75, \mathrm{NMRF}=4, \mathrm{LMRF}=1, \mathrm{EVRF}=0.04, \mathrm{kT}=0.3$. Corresponding radial average of power spectral density plot was shown at the bottom of each image.

Quantification of scaling symmetry in an image by power spectrum decay rate $(\beta)$ :

Consider a 2D greyscale or binary image as in Figure SVI-9a). The image is converted from spatial domain into frequency domain (wavevectors) using 2D Fourier transform. A surface plot of the estimated log power spectral density is shown in Figure SVI-9b). The lowest frequency component was centered (Figure SVI-9c)). The square of the real-valued component of the FT (Power $=$ Intensity $^{2}$, thus called 'Power Spectral Density, ${ }^{16}$ PSD, was calculated and transformed into polar coordinates. For each polar angle, power was plotted against frequency in a log-log scale. An average over the all possible angle gives the radial average of the power spectral density plot, as shown in Figure SVI-9d). For PSD based analysis, we measure the power-law exponent $\beta$, of an image. This is mostly done by linear fitting the log-log plot. Generally, when PSD is flat $(\beta=0)$, the signal is uncorrelated. ${ }^{17}$ While, images that contain features characterized by $\beta$ in the range, $1<\beta<3$, are considered as fractional Brownian motion, $\mathrm{fBM} .{ }^{17} \mathrm{fBM}$ is distinct from normal Brownian motion in a way that the random walk is not truly random here, therefore the step sizes are heterogeneous in space. Each move is influenced by its previous steps with a long-range correlation (memory), thus, produces a non-Merkovian framework for the particle dynamics. ${ }^{18}$ 
Apart from $\beta$, the appearance of peak in the PSD curve strongly suggests dominant spatial correlation in the image. ${ }^{19}$ The PSD curve is being noisy due to a lack of statistics, we rather calculate the discrete first moment from the data, which is defined as the probability-weighted
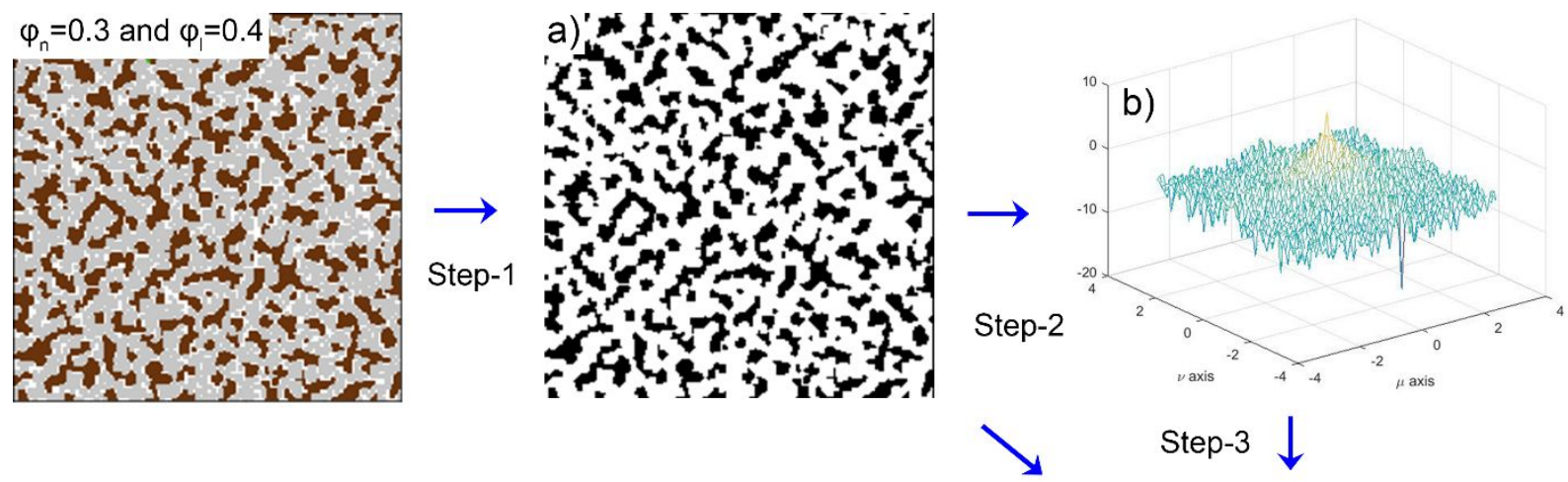

Step-3 $\downarrow$

First moment (Probability-weighted average of all raPSD values)

Reciprocal of first moment is the Characteristic length

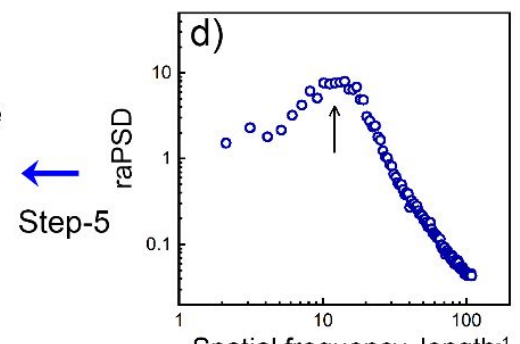

$\downarrow$ Step-6

Spatial frequency, length ${ }^{-1}$
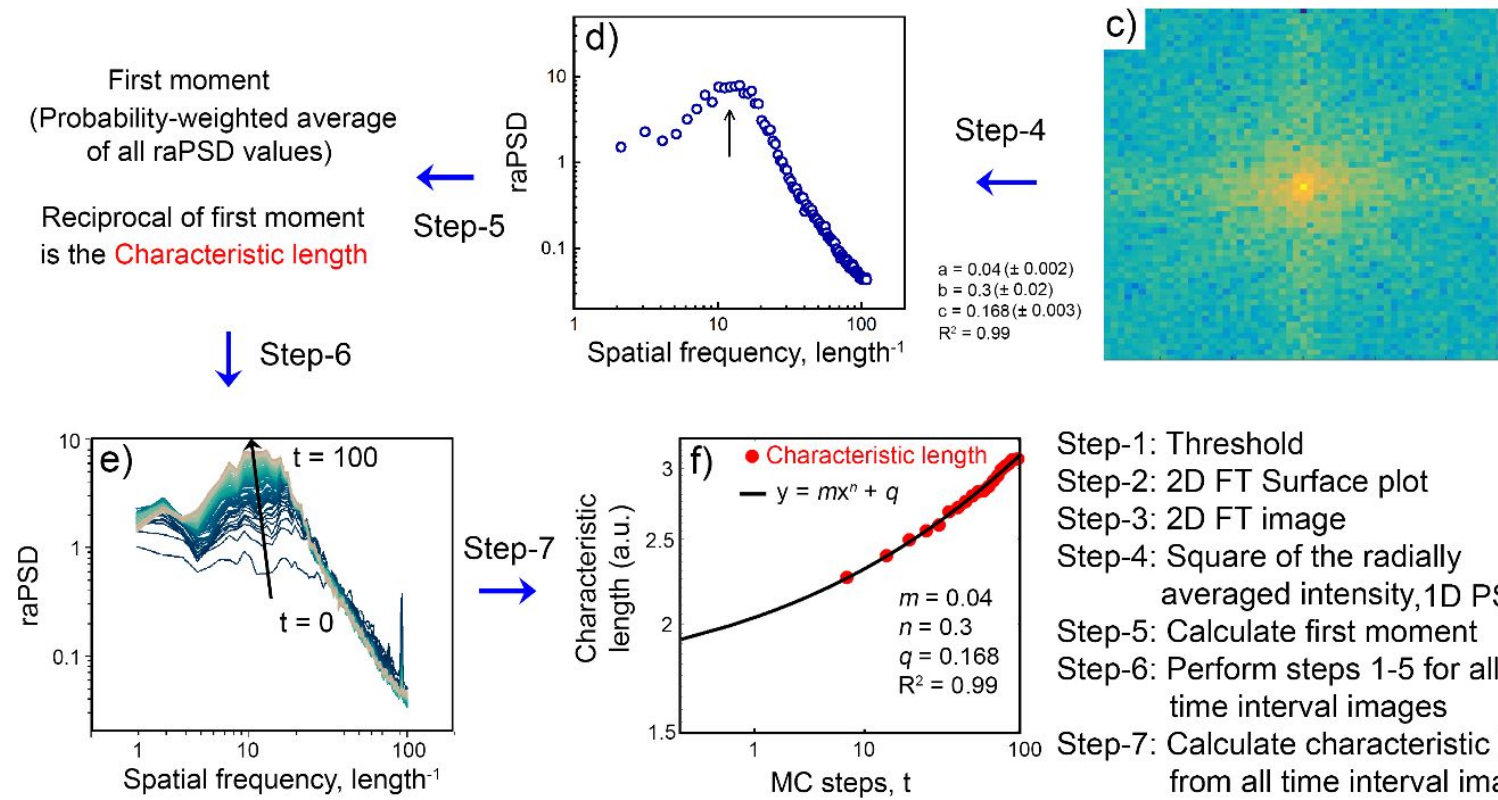

Step-1: Threshold

Step-2: 2D FT Surface plot

Step-3: 2D FT image

Step-4: Square of the radially averaged intensity, 1D PSD

Step-5: Calculate first moment

Step-6: Perform steps 1-5 for all time interval images

Step-7: Calculate characteristic length from all time interval images

Figure SVI-9: Step-by-step PSD analysis of a representative image from the final simulation. a) Threshold image of the original RGB image, crated to identify the scale length related to NP only. b) Surface plot of the Fourier transformed intensity, showing roughness of the surface related to the image. c) 2D PSD plot (phase value only), low frequency centered. d) Radial average of the 2D PSD in the spatial frequency range, plotted in log-log scale. e) Temporal evolution of the PSD curve generated from all time $(t=0$ to 100) interval images. $f)$ Plot of characteristic length with time, and growth law scaling of the process. Fitting parameters are shown in the image. Arrows are for guide to the eye. 
average, or the expected value of PSD. ${ }^{20}$ The characteristic length was calculated as the reciprocal of the first moment, corresponds to the average NP domain size. We have plotted the PSD curves from images taken at each time interval (i.e. after each MC step) in Figure SVI-9e). Characteristic lengths were calculated from each PSD curve and plotted with time in log-log scale in Figure SVI-9f) which gives the information about the temporal evolution of the NP patterning. The data was then fitted with a growth law equation of the type $m x^{n}+q$, (where $m=$ growth rate constant, $n=$ growth exponent, and $q=$ offset). The growth exponent was extracted from the fitting to be, $n=0.3$. A small offset was noticed, which is an artefact characterize to the initial randomization process. The value of the exponent matches well within the mentioned range in the literature $(0.1$ to 0.3$){ }^{21}$

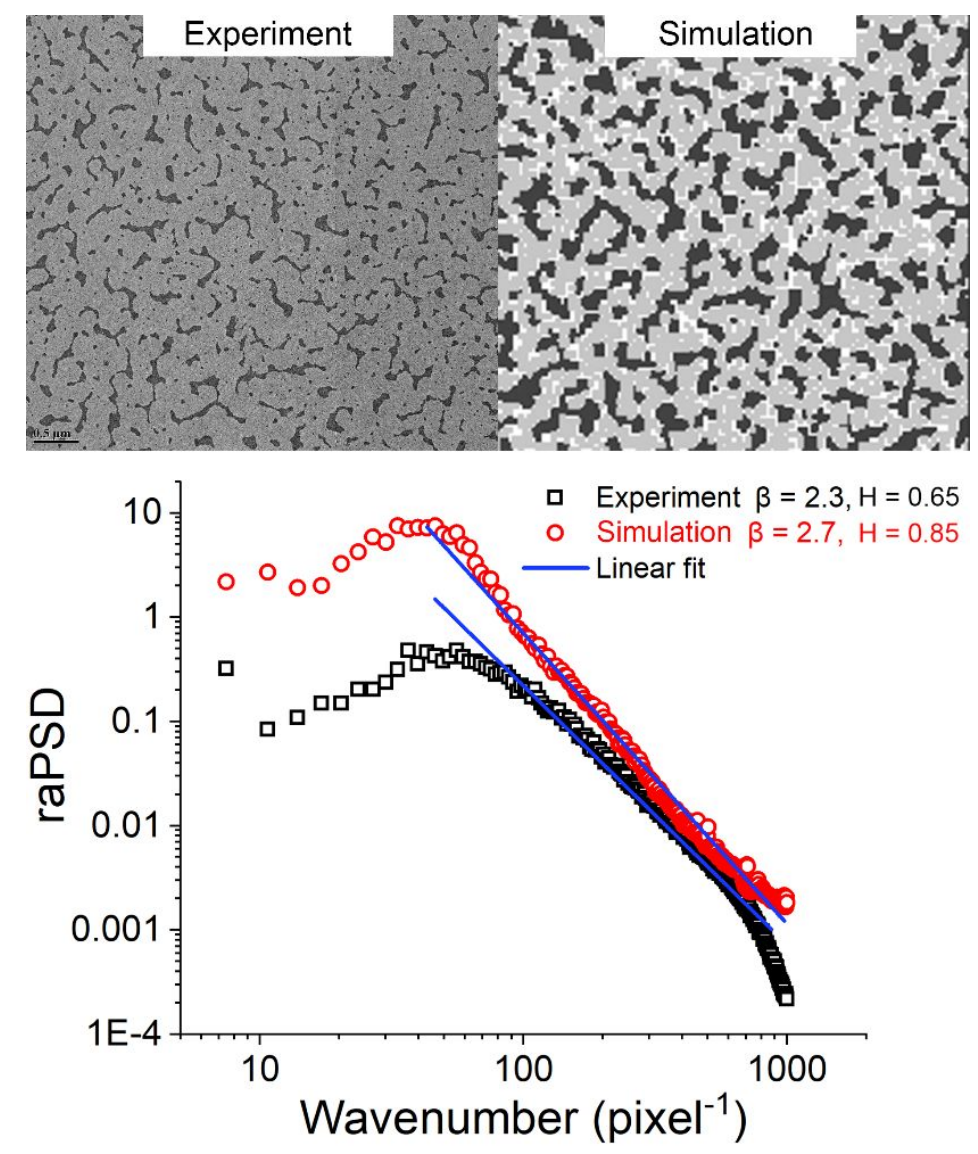

Figure SVI-10: Comparison of the power-law exponent $(\beta)$ and Hurst exponent $(\mathrm{H})$ from both the experimental and simulation studies using raPSD analysis without scaling the length. A reasonably good agreement between the obtained values may be noted. 
Appendix I: Numerical calculations

Calculation of ligand grafting density ( $\sigma$ ) from TGA:

Calculation of no. of ligand per particle from TGA:

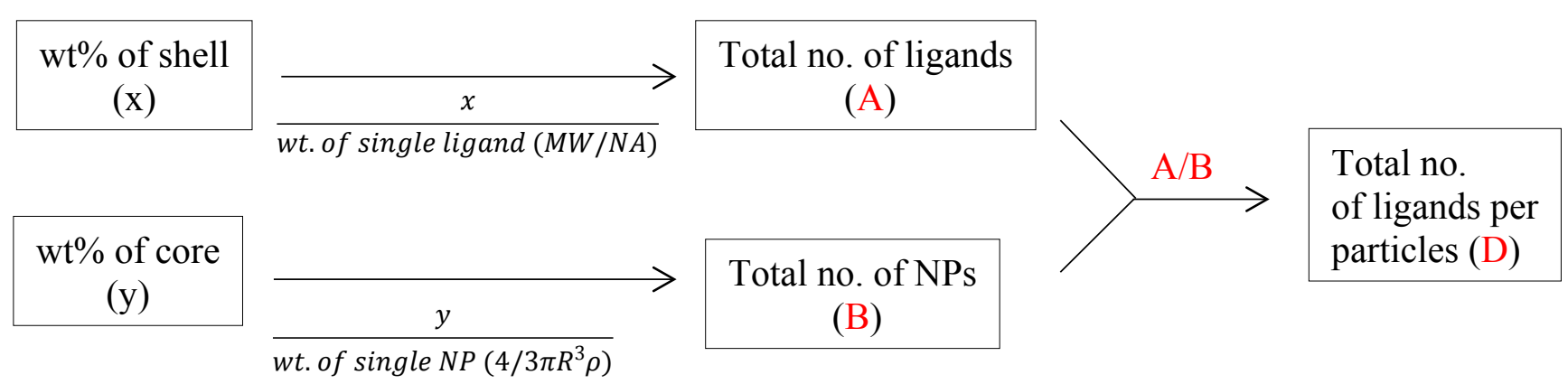

Calculation of ligand density per $\mathrm{nm}^{2}$ from TEM and TGA

$\begin{aligned} \begin{array}{c}\text { Particle radius (R) } \\ \text { from TEM }\end{array} & \stackrel{4 \pi R^{2}}{\longrightarrow} \begin{array}{c}\begin{array}{c}\text { Surface area } \\ \mathrm{S}\left(\mathrm{nm}^{2}\right)\end{array} \\ \sigma\end{array} \\ = & \left(\frac{w t \% \text { shell } \times N A \times \rho_{A u}^{\text {bulk }} \times \frac{4}{3} \pi R^{3}}{w t \% \text { core } \times M W_{\text {ligand }} \times 4 \pi R^{2} \times\left(10^{7}\right)^{3}}\right) / \mathrm{nm}^{2} \\ & =5.4 \text { ligands per } \mathrm{nm}^{2} \text { of particle }\end{aligned}$

Table 2: Chart for the calculation of ligand grafting density from TGA and TEM analysis.

\begin{tabular}{|c|c|c|c|c|c|}
\hline Ligand & wt\% (shell) & $\mathbf{w t} \%$ (core) & $\begin{array}{c}\text { MW of DDT } \\
\text { (g/mol) }\end{array}$ & $\mathbf{R}(\mathbf{n m})$ & $\boldsymbol{\sigma}\left(\mathbf{n m}^{-\mathbf{2}}\right)$ \\
\hline Thiol & 8.95 & 91.05 & 202.4 & 2.75 & 5.4 \\
\hline
\end{tabular}

\section{- Calculation of footprint area:}

Footprint area $\left(A_{o}^{\prime}=1 / \sigma\right)$ is the area occupied by a single ligand on the surface of the NP. However, it strongly depends on the ligand grafting density. Therefore, a correction factor is 
needed to the calculation which is defined by; Correction factor, $\left(\epsilon=\frac{A_{0}}{A_{o}^{\prime}}=\frac{0.23}{0.18}=1.277\right)$ as the ratio of nominal footprint area of the ligand $\left(A_{0}\right)$ to the area that the ligand actually occupies on the NP surface $\left(A_{0}^{\prime}\right) \cdot{ }^{22}$

\section{$\square$ Calculation of particle density in solution from ICP-AES:}

So, how many unit cells can be fitted within a single NP considering spherical shape of particle with radius, $\mathrm{R}=2.7 \mathrm{~nm}$ ?

No. of unit cell $=$ particle vol. $/$ unit cell vol. $=4 / 3 \pi \mathrm{R}^{3} / 0.068$

Each unit cell of Au contains 4 gold atoms

So, a single NP of radius, $R$, contains $4 / 3 \pi \mathrm{R}^{3} / 0.068 \times 4=4848$ gold atoms $(\mathrm{R}=2.7)$

No of spheres in original solution:

Gold atom concentration in the Au-DDT sample was back calculated to be $0.009 \mathrm{M}$.

$0.009 \mathrm{M} \mathrm{Au}$ solution contains $\sim 5 \times 10^{21} \mathrm{Au}$ atoms per $\mathrm{mL}$,

So, $0.009 \mathrm{M}$ Au solution contains total, $6.023 \times 10^{21} / 4848 \approx 10^{18}$ spheres per $\mathrm{mL}$,

We performed 100 times dilution of this solution for TEM imaging. Therefore, the particle density in the solution used for TEM analysis is $\sim 10^{16}$ per $\mathrm{mL}$.

\section{- Calculation of space filling property of NP array: ${ }^{22}$}

Optimal packing model (OPM): According to this model, ligands are densely packed only within the narrow volume along the overlapping space between the line connecting the centre of two NPs and predict an effective radius $(r)$ of the ligand coated NP, termed as disk, as, $r=R$ $\left(1+3 \epsilon \frac{L}{R}\right)^{\frac{1}{3}}$

The fraction of space occupied by the disk within the 2D pseudo-hcp lattice is the effectiveness of the packing. This can be calculated according to the following equation.

Packing efficiency $\left(\rho_{\text {disc }}^{h c p}\right)=\frac{N(\text { area taken up by the disk })}{\text { area of the unit cell }} ; \mathrm{N}=$ number of disks per unit cell $=3$

$$
=\frac{3 \times\left(A_{N P \text { core }}+A_{\text {ligand shell }}\right)}{A_{\text {unit cell }}^{\text {ch }}}=\frac{3 \times\left(\pi R^{2}+2 \pi R \sqrt{\sigma} A_{0} L_{e f f}\right)}{6 \sqrt{3} r^{2}}=64 \%
$$


The NP core and ligand shell contribution to the disc packing efficiency were calculated as,

$\rho_{\text {ligand }}^{\text {disc }}=\left(\frac{\text { total area of ligand shell }}{\text { area of unit cell }}\right)=\frac{3 \times\left(2 \pi R \sqrt{\sigma} A_{0} L_{e f f}\right)}{6 \sqrt{3} r^{2}}=\sim 20 \%$, and $\rho_{\text {core }}^{\text {disc }}=\left(\frac{\text { total area of NP core }}{\text { area of unit cell }}\right)=$

$\frac{3 \times \pi r^{2}}{6 \sqrt{3} r^{2}}=\sim 44 \%$. Rest $36 \%$ is empty space.

So, the space filling fraction of ligand shell to the voids of the unit cell can be calculated as, $\rho_{\text {ligand }}^{\text {void }}=\left(\frac{\text { total area of ligand shell }}{\text { area of unit cell }- \text { area of NP core }}\right)=\sim 36 \%$,

Similarly, space filling fraction of NP core to the voids of the unit cell can be calculated as, $\rho_{\text {core }}^{\text {void }}$ $=\left(\frac{\text { total area of NP core }}{\text { area of unit cell }- \text { area of ligand shell }}\right)=\sim 55 \%$.

\section{Section VII: References}

1) Mipar home page. https://www.mipar.us (accessed on June 7, 2019)

2) David, A.; Vassilvitskii, S. In K-means ${ }^{++}$: The advantages of careful seeding: SODA '07, Proceedings of the Eighteenth Annual ACM-SIAM Symposium on Discrete Algorithms. 2007, 1027-1035.

3) Lloyd, S. P. Least squares quantization in PCM. IEEE Trans. Inf. Theory 1982, 28, 129 137.

4) Bertilsson, L.; Liedberg, B. Infrared study of thiol monolayer assemblies on gold: preparation, characterization, and functionalization of mixed monolayers, Langmuir 1993, 9, 141-149.

5) Benoit, D. N.; Zhu, H.; Lilierose, M. H.; Verm, R. A.; Ali, N.; Morrison, A. N.; Fortner J. D.; Avendano, C.; Colvin, V. L. Measuring the grafting density of nanoparticles in solution by analytical ultracentrifugation and total organic carbon analysis. Anal. Chem. 2012, 84, 9238-9245.

6) Smith, A. M.; Johnston, K. A.; Crawford, S. E.; Marbella, L. E.; Millstone, J. E. Ligand density quantification on colloidal inorganic nanoparticles, Analyst 2017, 142, 11-29.

7) Hinterwirth, H.; Kappel, S.; Waitz, T.; Prohaska, T.; Lindner, W.; Lammerhofer, M. Quantifying thiol ligand density of self-assembled monolayers on gold nanoparticles by inductively coupled plasma-mass spectrometry. ACS Nano, 2013, 7, 1129-1136. 
8) Huhtamäki, T.; Tian, X.; Korhonen, J. T.; Ras, R. H. A. Surface-wetting characterization using contact-angle measurements. Nature Protocols 2018, 13, 2018, 1521-1538.

9) Chalmers, C. Modelling evaporation and phase behaviour of particle suspensions. Ph.D. thesis, Loughborough University, London, 2017.

10) Rabani, E.; Reichman, D. R.; Geissler, P. L.; Brus, L. E. Drying-mediated self-assembly of nanoparticles, Nature 2003, 426, 271-274.

11) Vancea, I.; Thiele, U.; Pauliac-Vaujour, E.; Stannard, A.; Martin, C. P.; Blunt, M. O.; Moriarty, P. J. Front instabilities in evaporatively dewetting nanofluids, Phys. Rev. E. 2008, 78, 041601.

12) Martin, C. P.; Blunt, M. O.; Pauliac-Vaujour, E.; Stannard, A.; Moriarty, P.; Vancea, I.; Thiele, U. Controlling pattern formation in nanoparticle assemblies via directed solvent dewetting. Phys. Rev. Lett. 2007, 99, 116103.

13) Metropolis, N.; Rosenbluth, A. W.; Rosenblutht, M. N.; Teller, A. H. Equation of state calculations by fast computing machines, J. Chem. Phys. 1953, 21, 1087-1092.

14) Bewerunge, J.; Ladadwa, I.; Platten, F.; Zunke, C.; Heuer, A.; Egelhaaf, S. U. Time- and ensemble-averages in evolving systems: the case of Brownian particles in random potentials, Phys. Chem. Chem. Phys. 2016, 18, 18887-18895.

15) Coropceanu, I.; Boles, M. A.; Talapin, D. V. Systematic mapping of binary nanocrystal superlattices: the role of topology in phase selection. J. Am. Chem. Soc. 2019, 141, 5728-5740.

16) Xylas, J.; Quinn, K. P.; Hunter, M.; Georgakoudi, I. Improved Fourier-based characterization of intracellular fractal features. Optics Express 2012, 20, 23442.

17) Turcotte, D. L. Fractals and chaos in geology and geophysics; Cambridge University Press: 1997.

18) Metzler, R.; Jeon, J-H.; Cherstvy, A. G.; Barkai, E. Anomalous diffusion models and their properties: non-stationarity, non-ergodicity, and ageing at the centenary of single particle tracking. Phys. Chem. Chem. Phys., 2014, 16, 24128.

19) Subramaniam, A. B.; Guidotti, G.; Manoharan, V. N.; Stone, H. A. Glycans pattern the phase behaviour of lipid membranes. Nat. Mater. 2013, 12, 128-133.

20) Teece, L. J.; Faers, M. A.; Bartlett, P. Aging and collapse in gel with ling-range attractions. Soft Matter 2011, 1, 7, 1341-1351. 
21) Ernst, H. J.; Fabre, F.; Lapujoulade, J., Observation of dynamical scaling in spinodal decomposition in two dimensions. Phys. Rev. Lett. 1992, 69, 458.

22) Boles, M. A.; Talapin, D. V. Many-body effects in nanocrystal superlattices: departure from sphere packing explains stability of binary phases. J. Am. Chem. Soc. 2015, 137, 4494-4502.

23) Evan Ruzanski, Radially averaged power spectrum of 2D real-valued matrix. 2013. Matlab central file exchange. 